\title{
Kinetic Equations for Fully Ionized, Inhomogeneous Plasmas
}

\author{
RICHARD K. OSBORN \\ Department of Nuclear Engineering, The University of Michigan, Ann Arbon, Michigan
}

(Received 28 January 1963)

\begin{abstract}
It is shown that, in the sense of certain procedures for successively approximating the quantum Liouville equation, the Boltzmann-Vlasov-Maxwell equations for the description of the fully ionized plasma are a natural first approximation. The nature of some of the approximations inherent in the use of such a description of the plasma is indicated, but little attention is given to their quantitative evaluation. An $H$ theorem for the particle-photon system is demonstrated.
\end{abstract}

\section{INTRODUCTION}

$T^{\prime}$ HE purpose of this paper is to present, from one particular point of view, a summary of some preliminary investigations of particle and photon transport in fully ionized gases. ${ }^{1-3}$ The emphasis on a particular point of view is not intended to suggest that it is necessarily the best vantage point from which to inspect the subject, but rather that it is a seemingly simplifying and clarifying, and yet so far, a somewhat unexploited vantage point. There is, however, one practical consideration which militates in favor of the approach to the plasma problem discussed below. As will be seen, at a certain level of approximation, the kinetic equations emerging naturally from the present treatment are the conventional Boltzmann-Vlasov equations for the particle distributions and Maxwell's equations for the electromagnetic fields. As these equations provide the basis for a large proportion of all attempts at a quantitive analysis of plasma behavior, it seems desirable that the foundations of these equations be investigated. In fact, it is precisely this issue that provides the primary motivation for this study. Furthermore, this emphasis means that no attempt shall be made herein to review the many interesting and varied approaches to this problem that have been developed in the past few years. ${ }^{4}$

Because recourse to experiment to test semi-intuitive models of the plasma is not often feasible, it seems necessary at the present time to investigate the validity (or range of approximate validity) of such models from strictly theoretical considerations. The accomplishment of such an objective requires, firstly, a comprehensive axiomatic statement of the problem (the axioms being reasonably widely agreed upon, of course), followed, secondly, by a deduction of descriptions of the plasma to which the various models purportedly correspond. Needless to say, no such ambitious program has yet been achieved.

\footnotetext{
${ }^{1}$ R. K. Osborn, University of Michigan Radiation Laboratory Report 02756-1-T, 1960, AF 33(616)-5585 (unpublished).

2 R. K. Osborn and E. H. Klevans, Ann. Phys. (N. Y.) 15, 105 (1961).

${ }^{3}$ R. K. Osborn, IRE Trans. Antennas Propagation 10, 8 (1962). Much of the content of the present article appears in this reference in abbreviated form. Furthermore, the present article also draws heavily from the University of Michigan Radiation Laboratory Technical Report No. 2764-8-T prepared by the author.

${ }^{4}$ For a considerable bibliography of investigations of the theory of plasmas consult, for example, J. Drummond, Plasma Physics (McGraw-Hill Book Company, Inc., New York, 1961).
}

The present discussion is restricted to the delineation of an approach to the problem of determining the validity of Boltzmann-type equations for the description of particle and photon balance in the fully ionized plasma. It is admitted at the outset that this approach essentially fails with respect to both of the main points indicated above. In the first place the selection of axioms is hardly universally agreed upon, and in the second place the deduction of consequences from the chosen axioms is far less rigorous than is desirable. Nevertheless, the results seem suggestive and represent somewhat of a generalization of the results usually discussed in the context of the present problem. Furthermore, though the deductions herein proceed via many approximations (none of which have been investigated in detail) the steps required for their testing are usually discernible.

The discussion is divided into several sections. Section I incorporates a statement of the axioms and some discussion thereof. Section II is devoted to an approximate deduction of a balance relation for the particles in the plasma and some consideration of Maxwell's equations. Section III presents a similar development of a transport equation for photons. In Sec. IV some of the implications of these balance relations for the thermodynamic state of the plasma are examined. In particular, an $H$ theorem for the particle-photon system is sketched.

\section{THE AXIOMS}

The axioms required for the description of systems of the type presently under discussion are usually considered to be of two kinds. The first of these is for the purpose of specifying the dynamics of the interactions between the particles that comprise the plasma, whereas the second is for the purpose of introducing statistical concepts into a description of a system characterized by a huge number of degrees of freedom. The dynamical axiom is conveniently expressed in terms of a Hamiltonian for the system; from which, according to the canonical equations, whether classically or quantum mechanically interpreted, all information may be deduced. Since we are here concerned with electrodynamics, we may expect that the dynamical axiom will be reasonably firm and noncontroversial at least within certain self-evident limitations such as, for example, nonrelativistic treatment of the particles. 
The statistical axiom is usually introduced via the concept of ensembles of systems, in terms of which the probability of finding the given system in a given state at a given instant can be meaningfully formulated. Though usually considered necessary (whether the system be dealt with in classical or quantum terms), we avoid the explicit introduction of such concepts into the present discussion. It is for this reason that our axiomatization of the system may be considered controversial, to say the least. Instead, we treat the system quantum mechanically and apparently rely solely upon the statistical concepts inherent in such a treatment. The equivocation is a recognition of the possibility that justification of some of the approximations to be invoked subsequently may require the ensemble conceptbut such a necessity is not evident at the moment. We note that all of the results of the conventional statistical treatments of systems similar to the one considered here are forthcoming from the present analysis.

The dynamical axiom is stated in terms of an energy density for fields of interacting charged particles and photons, and the Schrödinger equation for the wave function which characterizes the states of such a system. The field-theoretic formalism is dictated by the desire to deal with photon transport on the same footing as one deals with particle transport, and so far there has been no indication that this is feasible in either the classical or semiclassical context. ${ }^{5}$ It has the slight, further, formal advantage that the singlet densities whose equations we seek can be defined in terms of expectation values. In nondegenerate plasmas it is not expected that quantum effects play a significant role in the description of particle transport. In view of these remarks we have ${ }^{6}$

where

$$
H \Psi=i \hbar \partial \Psi / \partial t
$$

$$
H=\int_{\mathbf{x}} \mathfrak{H C}(\mathbf{x}) d^{3} x
$$

and

$$
\begin{aligned}
\mathcal{F}(\mathbf{x})= & -\sum_{\sigma} \frac{1}{2 m_{\sigma}}\left[\left(i \hbar \nabla-\frac{e_{\sigma}}{c} \mathbf{A}-\frac{e_{\sigma}}{c} \mathbf{A}^{\mathrm{e}}\right)_{j} \psi_{\sigma^{\dagger}}{ }^{\dagger}\right] \\
& \times\left[\left(i \hbar \boldsymbol{\nabla}+\frac{e_{\sigma}}{c} \mathbf{A}+\frac{e_{\sigma}}{c} \mathbf{A}^{e}\right)_{j} \psi_{\sigma}\right] \\
& +\left[2 \pi c^{2} \mathbf{P}^{2}+\frac{1}{8 \pi}(\boldsymbol{\nabla} \times \mathbf{A})^{2}\right]+\sum_{\sigma} e_{\sigma} \Phi \psi_{\sigma^{\dagger}} \psi_{\sigma} \\
& +\sum_{\sigma \sigma^{\prime}} \frac{e_{\sigma} e_{\sigma^{\prime}}}{2} \int d^{3} x \frac{\psi_{\sigma^{\dagger}}(\mathbf{x}) \psi_{\sigma^{\prime}}^{\dagger}\left(\mathbf{x}^{\prime}\right) \psi_{\sigma^{\prime}}\left(\mathbf{x}^{\prime}\right) \psi_{\sigma}(\mathbf{x})}{\left|\mathbf{x}-\mathbf{x}^{\prime}\right|} .
\end{aligned}
$$

In Eq. (3), $\psi_{\sigma}$ is a wave operator for a field of particles of the $\sigma$ th kind, $\mathbf{A}^{e}$ and $\Phi$ are the vector and scalar

\footnotetext{
${ }^{5}$ For classical, microscopic treatments of radiation in plasmas, see A. Simon and E. Harris, Phys. Fluids 3, 245, 255 (1960).

${ }^{6}$ L. I. Schiff, Quantum Mechanics (McGraw-Hill Book Company, Inc., New York, 1955), 2nd ed.
}

potentials of the "external fields," and $\mathbf{A}$ and $\mathbf{P}$ are the "transverse" magnetic and electric operators for the photon field. The external fields are presumed known and, hence, are unquantized, whereas the "internal fields" are described by operators which satisfy the commutation relations

$$
\left[\psi_{\sigma}(\mathbf{x}), \psi_{\sigma^{\prime}}{ }^{\dagger}\left(\mathbf{x}^{\prime}\right)\right]_{ \pm}=\delta_{\sigma \sigma^{\prime}} \delta\left(\mathbf{x}-\mathbf{x}^{\prime}\right)
$$

and

$$
\begin{aligned}
& {\left[A_{j}(\mathbf{x}), P_{i}(\mathbf{x})\right]_{-}} \\
& \quad=i \hbar \delta_{j i} \delta\left(\mathbf{x}-\mathbf{x}^{\prime}\right)-i \hbar \nabla_{j} \nabla_{i}\left(\frac{1}{4 \pi\left|\mathbf{x}-\mathbf{x}^{\prime}\right|}\right),
\end{aligned}
$$

all other quantities commuting or anticommuting. The particle field operators satisfy anticommutation or commutation rules depending upon whether they represent fermions or bosons. This distinction is expected to be of no importance for the nondegenerate plasma, but will be maintained for the sake of generality and because it introduces no appreciable complication. It should be noted in passing that we are treating all particle field operators as scalars and, hence, are introducing an inconsistency in principle whenever anticommutation rules are presumed applicable (as, for example, they are for the electrons). Such a treatment is hardly necessary, but entails so little loss of generality in the nonrelativistic treatment of the plasma that it seems warranted by the slight calculational simplification that it provides. Thus, spin effects will be absent here, e.g., forces exerted on electrons due to coupling of electron spins with inhomogeneous magnetic fields, ${ }^{7}$ and the spin dependence of cross sections for the scattering of identical particles. ${ }^{6}$

The so-called statistical axiom for the system is formulated in terms of the definitions of singlet densities for the particles and photons. Some difficulty (or at least deviousness) is to be anticipated here, since the classical concept of a distribution function implies arbitrarily precise localization of particles in phase space. A way out (and the one chosen here, as it has been many times elsewhere) is simply to give up the notion of "fine-grained meaningfulness" of the singlet densities. Alternatively, and somewhat equivalently, we may solely require of these singlet densities that they be suitable weight functions for the calculation of observable averages.

As an analytical tool for the implementation of the notion of a "coarse-grained" density, we shall adopt Ono's method of quantization in cells. ${ }^{8,9}$ Since the details of this procedure have been extensively discussed by $\mathrm{Ono}^{8}$ in his study of transport in neutral

\footnotetext{
${ }^{7}$ E. Ozizmir, Doctoral thesis, University of Michigan, 1962 (unpublished).

8 S. Ono, Progr. Theoret. Phys. (Kyoto) 12, 113 (1954).

${ }^{9} \mathrm{~S}$. Ono, in Proceedings of the International Symposium on Transport Processes in Statistical Mechanics (Interscience Publishers, Inc., New York, 1959), p. 229.
} 
gases, we shall merely sketch those aspects here which are necessary to the present application.

We introduce a complete set of cell functions defined as

where

$$
\varphi(\mathbf{X}, \mathbf{K}, \mathbf{x})=L^{-3 / 2} e^{i \mathbf{K} \cdot \mathbf{x}} E(\mathbf{X}, \mathbf{x})
$$

$$
E(\mathbf{X}, \mathbf{x})=\prod_{j=1}^{3} E\left(X_{j}, x_{j}\right)
$$

and

$$
\begin{aligned}
E\left(X_{j}, x_{j}\right) & =1, \quad \text { for } X_{j}-L / 2<x_{j}<X_{j}+L / 2 \\
& =\frac{1}{2}, \quad \text { for } x_{j}=X_{j} \pm L / 2 \\
& =0, \quad \text { otherwise. }
\end{aligned}
$$

Evidently $\mathbf{X}$ is a point centered in a cube of volume $L^{3}$, and we shall assume that all space has been subdivided into a regular, nonoverlapping array of such cubes. The variable $\mathbf{K}$ is also discretely distributed and is permitted the values stemming from the requirement that the exponential function be periodic on the boundaries of the cube, i.e.,

$$
K_{j}=2 \pi n_{j} / L, \quad n_{j}=0, \pm 1, \cdots .
$$

An arbitrary, measurable function defined over the domain of $\mathbf{x}$ may now be represented by a series of these functions as

$$
G(\mathbf{x})=\sum_{\mathbf{X}, \mathbf{K}} g(\mathbf{X}, \mathbf{K}) \varphi(\mathbf{X}, \mathbf{K}, \mathbf{x}),
$$

where the coefficients in the expansion are determined by

$$
g(\mathbf{X}, \mathbf{K})=\int d^{3} x \varphi^{*}(\mathbf{X}, \mathbf{K}, \mathbf{x}) G(\mathbf{x}) .
$$

The function set is orthonormal,

$$
\int d^{3} x \varphi^{*}(\mathbf{X}, \mathbf{K}, \mathbf{x}) \varphi\left(\mathbf{X}^{\prime}, \mathbf{K}^{\prime}, \mathbf{x}\right)=\delta_{\mathbf{X X}}{ }^{\prime} \delta_{\mathbf{K K}^{\prime}}
$$

and complete,

$$
\sum_{\mathbf{X}, \mathbf{K}} \varphi^{*}(\mathbf{X}, \mathbf{K}, \mathbf{x}) \varphi\left(\mathbf{X}, \mathbf{K}, \mathbf{x}^{\prime}\right)=\delta\left(\mathbf{x}-\mathbf{x}^{\prime}\right) .
$$

Consider the expansion of the field operator, $\psi_{\sigma}(\mathbf{x})$ in terms of these functions, i.e.,

$$
\begin{aligned}
\psi_{\sigma}(\mathbf{x}) & =\sum_{\mathbf{X}, \mathbf{K}} a_{\sigma}(\mathbf{X}, \mathbf{K}) \varphi(\mathbf{X}, \mathbf{K}, \mathbf{x}), \\
\psi_{\sigma}^{\dagger}(\mathbf{x}) & =\sum_{\mathbf{X}, \mathbf{K}} a_{\sigma}^{\dagger}(\mathbf{X}, \mathbf{K}) \varphi^{*}(\mathbf{X}, \mathbf{K}, \mathbf{x})
\end{aligned}
$$

The coefficients, $a_{\sigma}^{\dagger}(\mathbf{X}, \mathbf{K})$ and $a_{\sigma}(\mathbf{X}, \mathbf{K})$, are the creation and destruction operators for particles of kind $\sigma$ having momentum $\hbar \mathbf{K}$ in the cube centered at $\mathbf{X}$, respectively. They satisfy the usual commutation rules, e.g.,

$$
\left[a_{\sigma}(\mathbf{X}, \mathbf{K}), a_{\sigma}^{\dagger}\left(\mathbf{X}^{\prime}, \mathbf{K}^{\prime}\right)\right]_{ \pm}=\delta_{\mathbf{X X}^{\prime}} \delta_{\mathrm{KK}^{\prime}}
$$

The number operator for these particles is given by

$$
\rho_{\sigma}(\mathbf{X}, \mathbf{K})=a_{\sigma}^{\dagger}(\mathbf{X}, \mathbf{K}) a_{\sigma}(\mathbf{X}, \mathbf{K}) .
$$

We then define a distribution function for particles of the $\sigma$ th kind by

$$
f_{\sigma}(\mathbf{X}, \mathbf{K}, t)=L^{-3} \operatorname{Tr} \rho_{\sigma}(\mathbf{X}, \mathbf{K}) D(t),
$$

where $D(t)$ is the density matrix for the system and satisfies the equation

$$
\partial D / \partial t=(i / \hbar)[D, H] .
$$

The factor, $L^{-3}$, was included in the definition of $f_{\sigma}$ so that it would have the meaning of a volume average of the expected number of particles of kind $\sigma$ having momentum $\hbar \mathbf{K}$ in the cube centered at $\mathbf{X}$ at time $t$.

The distribution function for the photons is defined in a wholly analogous fashion. ${ }^{10}$ Let $\alpha_{\lambda}^{\dagger}(\mathbf{X}, \mathbf{k})$ and $\alpha_{\lambda}(\mathbf{X}, \mathbf{k})$ be the creation and destruction operators for photons of polarization $\lambda$ having momentum $\hbar \mathbf{k}$ in the cube about $\mathbf{X}$. Then

$$
\rho_{\lambda}(\mathbf{X}, \mathbf{K})=\alpha_{\lambda}{ }^{\dagger}(\mathbf{X}, \mathbf{K}) \alpha_{\lambda}(\mathbf{X}, \mathbf{K})
$$

is the number operator for these photons, and

$$
f_{\lambda}(\mathbf{X}, \mathbf{K}, t)=L^{-3} \operatorname{Tr} \rho_{\lambda}(\mathbf{X}, \mathbf{K}) D(t)
$$

is the definition of the corresponding distribution function. Of course, the creation and destruction operators for the photons obey boson commutation rules.

\section{BALANCE RELATIONS FOR THE PARTICLES}

Our prime concern in this section shall be for the deduction of a Boltzmann-type equation for the particle singlet density defined by Eq. (16). It is an implication of such an equation that the interactions between the particles of interest and the internal and external fields be treated in the sense of certain approximations in two extreme ways. On the one hand, the particles are thought of as being smoothly accelerated by the external fields and some suitable portion of the internal fields; and the effect of such a description of the interactions is to introduce the Vlasov-type terms into the equation for the time rate of change for the singlet density. The internal fields are dealt with self-consistently in that they are presumed governed by Maxwell's equations with internal currents and charge densities (calculable from the $f_{\sigma}$ 's) as sources. Thus, in some sense, this portion of the equation emphasizes collective behavior. On the other hand, the particles are also thought of as experiencing occasional impulsive forces from the remainder of the internal fields. This portion of these fields is dealt with in particulate terms (they are nearby charged particles in the case of the Coulomb field and photons in the case of the transverse electromagnetic fields), and the sense of the binary

${ }^{10}$ E. H. Klevans, Doctoral thesis, University of Michigan, 1962 (unpublished). 
interaction limit give rise to the collision terms in the balance equation for $f_{\sigma}$. Thus, this conception of the interactions emphasizes individual particle behavior. Self-consistency in this latter instance requires knowledge of distribution functions for all kinds of particles and for the photons.

Of course, such a subdivision of the interaction is artificial; nevertheless, it is intuitively pleasing in that it seems to provide a framework within which opposing extremes are simultaneously accounted for. It is hoped that the description is adequate between the extremes as well. Certainly the expenditure of a great deal of effort in the past several years has been predicated upon that hope. It is for this reason that the present viewpoint is being explored, i.e., the present approach to the problem lends itself naturally to the conception of self-consistent fields vs collisions at a certain order of approximation in the description of the singlet densities. To accommodate this notion, we display the Hamiltonian as

where

$$
H=T^{P}+V^{P},
$$

$$
\begin{aligned}
T^{P}= & L^{-3} \sum_{\sigma \mathbf{X} \mathbf{K X}^{\prime} \mathbf{K}^{\prime}} \frac{\hbar^{2}}{2 m_{\sigma}} a_{\sigma}^{\dagger}(\mathbf{X}, \mathbf{K}) a_{\sigma}\left(\mathbf{X}^{\prime}, \mathbf{K}^{\prime}\right) \int d^{3} x e^{i \mathbf{x} \cdot\left(\mathbf{K}^{\prime}-\mathbf{K}\right)} \\
& \times\left[i E\left(\mathbf{X}^{\prime}, \mathbf{x}\right) \mathbf{K}^{\prime} \cdot \nabla E(\mathbf{X}, \mathbf{x})-i E(\mathbf{X}, \mathbf{x}) \mathbf{K} \cdot \nabla E\left(\mathbf{X}^{\prime}, \mathbf{x}\right)+\nabla E(\mathbf{X}, \mathbf{x}) \cdot \nabla E\left(\mathbf{X}^{\prime}, \mathbf{x}\right)\right] \\
& +\sum_{\sigma} \frac{i \hbar e_{\sigma}}{m_{\sigma} c} \int d^{3} x \mathbf{R} \cdot \psi_{\sigma}^{\dagger} \nabla \psi_{\sigma}+\sum_{\sigma} \frac{e_{\sigma}^{2}}{2 m_{\sigma} c^{2}} \int d^{3} x R^{2} \psi_{\sigma}^{\dagger} \psi_{\sigma}+\sum_{\sigma} e_{\sigma} \int d^{3} x \Phi \psi_{\sigma}^{\dagger} \psi_{\sigma} \\
+ & \sum_{\sigma \sigma^{\prime}} \frac{e_{\sigma} e_{\sigma^{\prime}}}{2} \sum_{\mathbf{K} \mathbf{K}^{\prime} \mathbf{K}^{\prime \prime} \mathbf{K}^{\prime \prime \prime}} \sum_{\mathbf{X} \mathbf{X}^{\prime} ; \mathbf{X} \neq \mathbf{X}^{\prime}} a_{\sigma}^{\dagger}(\mathbf{X}, \mathbf{K}) a_{\sigma}\left(\mathbf{X}, \mathbf{K}^{\prime \prime \prime}\right) a_{\sigma^{\prime}}{ }^{\dagger}\left(\mathbf{X}^{\prime}, \mathbf{K}^{\prime}\right) a_{\sigma^{\prime}}\left(\mathbf{X}^{\prime}, \mathbf{K}^{\prime \prime}\right) \int \frac{d^{3} x d^{3} x^{\prime}}{\left|\mathbf{x}-\mathbf{x}^{\prime}\right|} \varphi^{*}(\mathbf{X}, \mathbf{K}, \mathbf{x}) \varphi\left(\mathbf{X}, \mathbf{K}^{\prime \prime \prime}, \mathbf{x}\right) \\
& \times \varphi^{*}\left(\mathbf{X}^{\prime}, \mathbf{K}^{\prime}, \mathbf{x}\right) \varphi\left(\mathbf{X}^{\prime}, \mathbf{K}^{\prime \prime}, \mathbf{x}^{\prime}\right),
\end{aligned}
$$

and

$$
\begin{aligned}
& V^{P}= \sum_{\sigma} \sum_{\mathbf{X}, \mathbf{K}} \frac{\hbar^{2} K^{2}}{2 m_{\sigma}} \rho_{\sigma}(\mathbf{X}, \mathbf{K})+\sum_{\sigma} \frac{i \hbar e_{\sigma}}{m_{\sigma} c} \int d^{3} x \mathbf{A}^{f} \cdot \psi_{\sigma}^{\dagger} \nabla \psi_{\sigma}+\sum_{\sigma} \frac{e_{\sigma}^{2}}{2 m_{\sigma} c^{2}} \int d^{3} x\left(\mathbf{A}^{f} \cdot \mathbf{R}+\mathbf{R} \cdot \mathbf{A}^{f}\right) \psi_{\sigma}^{\dagger} \psi_{\sigma} \\
&+\sum_{\sigma} \frac{e_{\sigma}^{2}}{2 m_{\sigma} c^{2}} \int d^{3} x \mathbf{A}^{f} \cdot \mathbf{A}^{f} \psi_{\sigma}^{\dagger} \psi_{\sigma}+\int d^{3} x\left[2 \pi c^{2} P^{2}+\frac{1}{8 \pi}(\nabla \times \mathbf{A})^{2}\right] \\
&+\sum_{\sigma \sigma^{\prime}} \frac{e_{\sigma} e_{\sigma^{\prime}}}{2} \sum_{\mathbf{X K K ^ { \prime } \mathbf { K } ^ { \prime \prime } \mathbf { K } ^ { \prime \prime \prime }}} a_{\sigma}^{\dagger}(\mathbf{X}, \mathbf{K}) a_{\sigma^{\prime}}{ }^{\dagger}\left(\mathbf{X}, \mathbf{K}^{\prime}\right) a_{\sigma^{\prime}}\left(\mathbf{X}, \mathbf{K}^{\prime \prime}\right) a_{\sigma}\left(\mathbf{X}, \mathbf{K}^{\prime \prime \prime}\right) \int \frac{d^{3} x d^{3} x^{\prime}}{\left|\mathbf{x}-\mathbf{x}^{\prime}\right|} \varphi^{*}(\mathbf{X}, \mathbf{K}, \mathbf{x}) \varphi\left(\mathbf{X}, \mathbf{K}^{\prime \prime \prime}, \mathbf{X}\right) \\
& \times \varphi^{*}\left(\mathbf{X}, \mathbf{K}^{\prime}, \mathbf{x}^{\prime}\right) \varphi\left(\mathbf{X}, \mathbf{K}^{\prime \prime}, \mathbf{x}^{\prime}\right) .
\end{aligned}
$$

Here we have divided the vector potential of the internal electromagnetic field into two parts of "fast" and "slow" variation respectively, i.e.,

$$
\mathbf{A}=\mathbf{A}^{f}+\mathbf{A}^{s}
$$

and then introduced

$$
\mathbf{R}=\mathbf{A}^{s}+\mathbf{A}^{e} .
$$

Thus, $\mathbf{R}$ is a superposition of that portion of the internal electromagnetic field generated by collective particle behavior and the external field. Crudely, $\mathbf{A}^{s}$ is of slow variation in the sense that it does not vary appreciably in space over dimensions of order $L$. The electric and magnetic fields associated with $\mathbf{R}$ give rise to the forces producing smooth accelerations of the particles under observation, whereas $\mathbf{A}^{f}$ is the part of the internal field to be treated as a photon field. The terms in the Hamiltonian representing the kinetic and Coulomb energies of the particles have been expanded into the basis vectors (5) and pieces assigned variously to $T^{P}$ and $V^{P}$. The clue to the assignment lies in the fact that $T^{P}$ is intended (in lowest order) to describe temporal variations of the density due to transport and smooth forces whereas $V^{P}$ is intended to describe the influence of collisions. Thus, $\mathbf{R}$ appears mainly in $T^{P}$ as does that part of the Coulomb energy arising from the Coulomb interactions between particles in a given cell with other particles outside that cell. Conversely, $\mathbf{A}^{f}$ appears mainly in $V^{P}$ along with the Coulomb energy associated with particles in the same cell. Note that that part of the kinetic energy which is proportional to $\rho_{\sigma}(\mathbf{X}, \mathbf{K})$ is incorporated into $V^{P}$. This is because in the present treatment these terms give rise to no time variation of $f_{\sigma}$ but rather define the energies of the states between which particles jump during collisions.

A word of caution here. No attempt should be made to give a physical interpretation to the various terms arising from the expansion of parts of the Hamiltonian 
in terms of the cell functions. This expansion should be regarded as a purely formal procedure employed solely for the purpose of facilitating subsequent calculations. Now consider

$$
f_{\sigma}(\mathbf{X}, \mathbf{K}, t+s)=L^{-3} \operatorname{Tr} \rho_{\sigma}(\mathbf{X}, \mathbf{K}) D(t+s),
$$

where " $s$ " is some small, but finite (thus, we are "coarse-graining" in time also) time interval. The bounds on this time interval will be at least partially illuminated below, but at the moment we may surmise that it is small compared to the time required for a typical particle described by $f_{\sigma}$ to cross a cell and large compared to collision times. By virtue of Eq. (17) we have

$$
D(t+s)=U(s) D(t) U^{\dagger}(s),
$$

where $U$ is a unitary transformation defined by the equation

$$
i \hbar \partial U / \partial t=H U
$$

and the boundary condition,

$$
U(0)=I .
$$

In line with the above remarks on the separation of the temporal variation of $f_{\sigma}$ into parts arising from collective and individual particle behavior, we set

where $\zeta$ is defined by

$$
U=\zeta \Gamma
$$

$$
i \hbar \partial \zeta / \partial t=T^{P \zeta}, \quad \zeta(0)=I .
$$

Then according to $(27)$

$$
i \hbar \partial \Gamma / \partial t=\zeta^{\dagger} V^{P} \zeta \Gamma
$$

and according to (28),

$$
\Gamma(0)=I
$$

also. Both $\zeta$ and $\Gamma$ are unitary. Equation (25) may now be written as

$f_{\sigma}(\mathbf{X}, \mathbf{K}, t+s)$

$$
=L^{-3} \operatorname{Tr} \zeta^{\dagger}(s) \rho_{\sigma}(\mathbf{X}, \mathbf{K}) \zeta(s) \Gamma(s) D(t) \Gamma^{\dagger}(s) .
$$

If the external fields were not time-dependent we would have

$$
\zeta(s)=e^{-i s T^{P} / \hbar}
$$

Since, in general, they are, the solution (34) is only approximate, but will be a good approximation if they do not vary appreciably over time intervals of the order of "s." That this is the case will be assumed in the following. The factor $\zeta^{\dagger} \rho_{\sigma} \zeta$ is expanded in a power series in " $s$ " and only the zeroth-and first-order terms retained, yielding

$$
\begin{aligned}
& f_{\sigma}(\mathbf{X}, \mathbf{K}, t+s) \\
& \simeq L^{-3} \operatorname{Tr}(i s / \hbar)\left[T^{P}, \rho_{\sigma}(\mathbf{X}, \mathbf{K})\right] \Gamma(s) D(t) \Gamma^{\dagger}(s) \\
&+L^{-3} \operatorname{Tr} \rho_{\sigma}(\mathbf{X}, \mathbf{K}) \Gamma(s) D(t) \Gamma^{\dagger}(s) .
\end{aligned}
$$

Because of (32), the first term on the right-hand side may be further approximated by neglecting the $s$ de- pendence of $\Gamma$. We then have

$$
\begin{aligned}
f_{\sigma}(\mathbf{X}, \mathbf{K}, t+s)-L^{-3} & \operatorname{Tr}(i s / \hbar)\left[T^{P}, \rho_{\sigma}(\mathbf{X}, \mathbf{K})\right] D(t) \\
& \simeq L^{-3} \operatorname{Tr} \rho_{\sigma}(\mathbf{X}, \mathbf{K}) \Gamma(s) D(t) \Gamma^{\dagger}(s) .
\end{aligned}
$$

In a representation which diagonalizes the operator $\rho_{\sigma}$, the right-hand side of Eq. (36) becomes

$$
\begin{aligned}
& L^{-3} \operatorname{Tr} \rho_{\sigma} \Gamma D \Gamma^{\dagger} \\
& \quad=L^{-3} \sum_{n n^{\prime}} \rho_{\sigma n n}\left|\Gamma_{n n^{\prime}}\right|{ }^{2} D_{n n^{\prime}}+\text { (terms proportional }
\end{aligned}
$$$$
\text { to off-diagonal elements of } D \text { ). }
$$

We will ignore the contribution of the terms involving the off-diagonal elements of the density matrix. In such an event Eq. (37) becomes

$$
\begin{aligned}
& L^{-3} \operatorname{Tr} \rho_{\sigma} \Gamma D \Gamma^{\dagger} \simeq L^{-3} \sum_{n} \rho_{\sigma n n}\left|\Gamma_{n n}\right|{ }^{2} D_{n n} \\
& +L^{-3} \sum_{n n^{\prime}}^{\prime} \rho_{\sigma n n}\left|\Gamma_{n n^{\prime}}\right|^{2} D_{n^{\prime} n^{\prime}}
\end{aligned}
$$

where the prime on the sum symbol implies that terms for which $n^{\prime}=n$ are to be omitted from the sum. By virtue of the unitarity of $\Gamma$ we have

$$
\left|\Gamma_{n n}\right|^{2}=1-\sum_{n^{\prime}}\left|\Gamma_{n^{\prime} n}\right|^{2}
$$

so that (38) may again be rewritten as

$$
\begin{aligned}
L^{-3} \operatorname{Tr} \rho_{\sigma} \Gamma D \Gamma^{\dagger} \simeq & f_{\sigma}(\mathbf{X}, \mathbf{K}, t) \\
& +L^{-3} \sum_{n n^{\prime}}\left(\rho_{\sigma n^{\prime} n^{\prime}}-\rho_{\sigma n n}\right)\left|\Gamma_{n n^{\prime}}\right|^{2} D_{n n} .
\end{aligned}
$$

Entering (40) into (36) and dividing by $s$, we obtain

$$
\begin{aligned}
\frac{f_{\sigma}(\mathbf{X}, \mathbf{K}, t+s)-f_{\sigma}(\mathbf{X}, \mathbf{K}, t)}{s}-L^{-3} \underset{\hbar}{\operatorname{Tr}-\left[T^{P}, \rho_{\sigma}(\mathbf{X}, \mathbf{K})\right] D(t)} \\
\simeq L^{-3} \sum_{n n^{\prime}}\left(\rho_{\sigma n^{\prime} n^{\prime}}-\rho_{\sigma n n}\right) \mathcal{T}_{n^{\prime} n} D_{n n}, \quad(41)
\end{aligned}
$$

where we have defined

$$
\mathcal{T}_{n^{\prime} n} \equiv(1 / s)\left|\Gamma_{n^{\prime} n}\right|^{2} .
$$

The evaluation to be presented here of the commutator in Eq. (41) is quite straightforward, though tedious and subject to several approximations. Hence, we shall merely give the result and discuss the approximations. With the identification of the first term on the left-hand side of (41) as the partial time derivative of the density, we obtain

$$
\begin{aligned}
& \frac{\partial f_{\sigma}}{\partial t}+\left(\frac{P_{j}}{m_{\sigma}}-\frac{e_{\sigma}}{m_{\sigma} c}\left\langle R_{j}\right\rangle\right) \frac{\partial f_{\sigma}}{\partial X_{j}} \\
& +\left(\frac{e_{\sigma}}{m_{\sigma} c} P_{l} \frac{\partial\left\langle R_{l}\right\rangle}{\partial X_{j}}-\frac{e_{\sigma}^{2}}{2 m_{\sigma} c^{2}} \frac{\partial\langle\mathbf{R}\rangle^{2}}{\partial X_{j}}-e_{\sigma} \frac{\partial \Phi}{\partial X_{j}}-e_{\sigma} \frac{\partial v}{\partial X_{j}}\right) \frac{\partial f_{\sigma}}{\partial P_{j}} \\
& =L^{-3} \sum_{n n^{\prime}}\left(\rho_{\sigma n^{\prime} n^{\prime}}-\rho_{\sigma n n}\right) \mathcal{T}_{n^{\prime} n} D_{n n}
\end{aligned}
$$


Here we have introduced the momentum, $\mathbf{P}=\hbar \mathbf{K}$ and the symbol, $v$, to represent the electrostatic potential in the cell about $\mathbf{X}$ due to all charged particles outside of that cell, i.e.,

$$
\begin{aligned}
v(\mathbf{X})=\sum_{\sigma} e_{\sigma} \int \frac{d^{3} x}{|\mathbf{x}-\mathbf{X}|}[1-E(\mathbf{X}, \mathbf{x})] & \\
& \times \operatorname{Tr} \psi_{\sigma}^{\dagger}(\mathbf{x}) \psi_{\sigma}(\mathbf{x}) D .
\end{aligned}
$$

Because of the gradients appearing in the expression for $T^{P}$, one finds contributions to Eq. (43) which are roughly interpretable as describing a flow of particles between cells accompanied by momentum changes which are independent of the forces acting on the particles. More accurately, these terms arise as a consequence of the localization of the particles in regions of dimension $L$, which introduces an uncertainty in their momentum of order $\hbar / L$. These terms have been ignored, and, therefore, Eq. (43) is valid only for those densities representing particles with momenta large compared to $\hbar / L$, i.e., particles with De Broglie wavelengths small compared to $L$. Another approximation implicit in Eq. (43) is the replacement of products of averages by averages of products. This approximation is standard in the derivation of the Vlasov equation, which indeed is what we have if we set the right-hand side of
(43) equal to zero. Finally, we have further approximated here by the assumption that the fields, $\mathbf{R}, \Phi$, and $v$ are all sufficiently slowly varying in space that within a given cell they may be Taylor expanded about the center of the cell and only the first two terms retained. This assumption has also been made with respect to the density itself.

The reduction of the right-hand side of Eq. (43) to more or less familiar terms requires first of all a calculation of the elements of the matrix, $\tau$. The analysis required to this end is summarized in Eqs. (42), (32), (31), (22), and (21). A desirable first step is the rewriting of Eq. (22) in more immediately useful and directly interpretable terms. Introducing the decomposition, $\mathbf{P}=P^{f}+P^{s}$, and the expansions,

$$
\begin{aligned}
& \mathbf{A}^{f}=\sum_{\mathbf{X} \mathbf{k} \lambda}\left(\frac{2 \pi \hbar c}{k}\right)^{1 / 2} \varphi^{*}(\mathbf{X}, \mathbf{k}, \mathbf{x}) \zeta_{\lambda^{\dagger}}(\mathbf{X}, \mathbf{k}), \\
& \mathbf{P}^{f}=i \sum_{\mathbf{X} \mathbf{k} \lambda}\left(\frac{\hbar k}{8 \pi c}\right)^{1 / 2} \varphi^{*}(\mathbf{X}, \mathbf{k}, \mathbf{x}) \zeta_{\lambda}-(\mathbf{X}, \mathbf{k}),
\end{aligned}
$$

one finds that after considerable rearrangement (22) becomes

where

$$
\begin{aligned}
& \mathcal{E}=\sum_{\mathbf{X} \mathbf{k} \lambda} \frac{\hbar c k}{2}+\sum_{\mathbf{X} \mathbf{k} \lambda} \hbar c k \rho_{\lambda}(\mathbf{X}, \mathbf{k})+\sum_{\sigma \mathbf{X K}}\left[\frac{\hbar^{2} K^{2}}{2 m_{\sigma}}+\sum_{\lambda \mathbf{k}} \frac{\pi \hbar e_{\sigma}{ }^{2}}{m_{\sigma} c L^{3} k}\right] \rho_{\sigma}(\mathbf{X}, \mathbf{K})+\sum_{\sigma \lambda \mathbf{X} \mathbf{k}}(\hbar c k) \frac{2 \pi e_{\sigma}{ }^{2}}{m_{\sigma} c^{2} k^{2} L^{3}} \rho_{\sigma}(\mathbf{X}) \boldsymbol{\rho}_{\lambda}(\mathbf{X}, \mathbf{k}), \\
& T^{\gamma}=\sum_{\mathbf{X} \mathbf{k} \lambda \mathbf{X}^{\prime} \mathbf{k}^{\prime} \boldsymbol{\lambda}^{\prime}} \frac{\hbar c}{4\left(k k^{\prime}\right)^{1 / 2}} \int d^{3} x \varphi^{*}(\mathbf{X}, \mathbf{k}, \mathbf{x}) \varphi\left(\mathbf{X}^{\prime}, \mathbf{k}^{\prime}, \mathbf{x}\right)\left\{i\left[\mathbf{\Delta}(\mathbf{X}, \mathbf{x}) \times \zeta_{\lambda}+(\mathbf{X}, \mathbf{k})\right] \cdot\left[\mathbf{k}^{\prime} \times \zeta_{\lambda^{\prime}}+\left(\mathbf{X}^{\prime},-\mathbf{k}^{\prime}\right)\right]\right. \\
& \left.-i\left[\mathbf{k} \times \zeta_{\lambda}+(\mathbf{X}, \mathbf{k})\right] \cdot\left[\mathbf{\Delta}\left(\mathbf{X}^{\prime}, \mathbf{x}\right) \times \zeta_{\lambda^{\prime}}+\left(\mathbf{X}^{\prime},-\mathbf{k}^{\prime}\right)\right]+\left[\mathbf{\Delta}(\mathbf{X}, \mathbf{x}) \times \zeta_{\lambda}+(\mathbf{X}, \mathbf{k})\right] \cdot\left[\mathbf{\Delta}\left(\mathbf{X}^{\prime}, \mathbf{x}\right) \times \zeta_{\lambda^{\prime}}+\left(\mathbf{X}^{\prime},-\mathbf{k}^{\prime}\right)\right]\right\}, \\
& V^{C}=\sum_{\sigma \sigma^{\prime} \mathbf{X}} \sum_{\mathbf{K K} \mathbf{K}^{\prime \prime} \mathbf{K}^{\prime \prime \prime}} \frac{e_{\sigma} e_{\sigma^{\prime}}}{2} a_{\sigma}^{\dagger}(\mathbf{X}, \mathbf{K}) a_{\sigma^{\prime}}{ }^{\dagger}\left(\mathbf{X}, \mathbf{K}^{\prime}\right) a_{\sigma^{\prime}}\left(\mathbf{X}, \mathbf{K}^{\prime \prime}\right) a_{\sigma}\left(\mathbf{X}, \mathbf{K}^{\prime \prime \prime}\right) \\
& \times \int \frac{d^{3} x d^{3} x^{\prime}}{\left|\mathbf{x}-\mathbf{x}^{\prime}\right|} \varphi^{*}(\mathbf{X}, \mathbf{K}, \mathbf{x}) \varphi^{*}\left(\mathbf{X}, \mathbf{K}^{\prime}, \mathbf{x}^{\prime}\right) \varphi\left(\mathbf{X}, \mathbf{K}^{\prime \prime}, \mathbf{x}^{\prime}\right) \varphi\left(\mathbf{X}, \mathbf{K}^{\prime \prime \prime}, \mathbf{x}\right), \\
& g=-\sum_{\sigma \lambda \mathbf{X k K K}} \frac{\hbar e_{\sigma}(2 \pi \hbar c)^{1 / 2}}{m_{\sigma} c k^{1 / 2} L^{3 / 2}} \mathbf{K}^{\prime} \cdot \zeta_{\lambda}{ }^{+}(\mathbf{X}, \mathbf{k}) a_{\sigma}^{\dagger}(\mathbf{X}, \mathbf{K}) a_{\sigma}\left(\mathbf{X}, \mathbf{K}^{\prime}\right) \delta\left(\mathbf{K}^{\prime}-\mathbf{K}-\mathbf{k}\right), \\
& \mathcal{S}=\sum_{\sigma \lambda \lambda^{\prime} \mathbf{X}} \sum_{\mathbf{k} \mathbf{k}^{\prime} \mathbf{K} \mathbf{K}^{\prime} ; \mathbf{k} \neq \mathbf{k}^{\prime}} \frac{\pi \hbar e_{\sigma}^{2}}{m_{\sigma} c L^{3}\left(k k^{\prime}\right)^{1 / 2}} \zeta_{\lambda}+(\mathbf{X}, \mathbf{k}) \cdot \zeta_{\lambda^{\prime}}+\left(\mathbf{X},-\mathbf{k}^{\prime}\right) a_{\sigma}^{\dagger}(\mathbf{X}, \mathbf{k}) a_{\sigma}\left(\mathbf{X}, \mathbf{k}^{\prime}\right) \delta\left(\mathbf{K}^{\prime}-\mathbf{K}-\mathbf{k}+\mathbf{k}^{\prime}\right), \\
& \chi=\sum_{\sigma \lambda \mathbf{k X}} \frac{\pi \hbar e_{\sigma}{ }^{2}}{m_{\sigma} c k L^{3}} \boldsymbol{\varepsilon}_{\lambda}(\mathbf{k}) \cdot \boldsymbol{\varepsilon}_{\lambda}(-\mathbf{k})\left[\alpha_{\lambda}^{\dagger}(\mathbf{X}, \mathbf{k}) \alpha_{\lambda}{ }^{\dagger}(\mathbf{X},-\mathbf{k})+\alpha_{\lambda}(\mathbf{X},-\mathbf{k}) \alpha_{\lambda}(\mathbf{X}, \mathbf{k})\right] \rho_{\sigma}(\mathbf{X}) \\
& +\sum_{\sigma \mathbf{X} \mathbf{k} \mathbf{K K} \mathbf{K}^{\prime}} \frac{i \hbar e_{\sigma}(2 \pi \hbar c)^{1 / 2}}{m_{\sigma} c k^{1 / 2} L^{9 / 2}} a_{\sigma}^{\dagger}(\mathbf{X}, \mathbf{K}) a_{\sigma}\left(\mathbf{X}, \mathbf{K}^{\prime}\right) \zeta_{\lambda}+(\mathbf{X}, \mathbf{k}) \cdot \int d^{3} x \mathbf{\Delta}(\mathbf{X}, \mathbf{X}) e^{i \mathbf{X} \cdot\left(\mathbf{K}^{\prime}-\mathbf{K}-\mathbf{k}\right)} \\
& +\sum_{\sigma} \frac{e_{\sigma}^{2}}{2 m_{\sigma} c^{2}} \int d^{3} x\left(\mathbf{A}^{f} \cdot \mathbf{R}+\mathbf{R} \cdot \mathbf{A}^{f}\right) \psi_{\sigma}^{\dagger} \psi_{\sigma}+\int d^{3} x\left[2 \pi c^{2}\left\{\mathbf{P}^{f} \cdot \mathbf{P}^{s}+\mathbf{P}^{s} \cdot \mathbf{P}^{f}+\left(\mathbf{P}^{s}\right)^{2}\right\}\right. \\
& \left.+\frac{1}{8 \pi}\left\{\left(\boldsymbol{\nabla} \times \mathbf{A}^{f}\right) \cdot\left(\boldsymbol{\nabla} \times \mathbf{A}^{s}\right)+\left(\boldsymbol{\nabla} \times \mathbf{A}^{s}\right) \cdot\left(\boldsymbol{\nabla} \times \mathbf{A}^{f}\right)+\left(\boldsymbol{\nabla} \times \mathbf{A}^{s}\right)^{2}\right\}\right] .
\end{aligned}
$$


In these expressions we have introduced

$$
\begin{aligned}
\zeta_{\lambda}^{ \pm}(\mathbf{X}, \mathbf{k}) & =\alpha_{\lambda}^{\dagger}(\mathbf{X}, \mathbf{k}) \varepsilon_{\lambda}(\mathbf{k}) \pm \alpha_{\lambda}(\mathbf{X},-\mathbf{k}) \boldsymbol{\varepsilon}_{\lambda}(-\mathbf{k}) \\
\rho_{\sigma}(\mathbf{X}) & =\sum_{\mathbf{K}} \rho_{\sigma}(\mathbf{X}, \mathbf{K})
\end{aligned}
$$

and

$$
\Delta_{j}(\mathbf{X}, \mathbf{x})=2\left[\delta\left(x_{j}-X_{j}+\frac{1}{2} L\right)-\delta\left(x_{j}-X_{j}-\frac{1}{2} L\right)\right] .
$$

The $\boldsymbol{\varepsilon}_{\lambda}$ 's are the unit, orthogonal polarization vectors of the photon field.

The first term in the decompositon of $V^{P}$ consists of the infinite zero-point energy of the photon field (which will henceforth be ignored), the energy of the photons which is proportional to the photon density only, the energy of the particles (including a self-energy due to interaction with the radiation field which will also be ignored) which is proportional to the particle density only, and an energy proportional to both the particle and photon densities - but only the spatial density of the particles. This last term is perhaps most conveniently incorporated into the energy of the "free" photons with consequences to be touched on later and discussed elsewhere. ${ }^{2,10}$ In the calculation of the right-hand side of Eq. (43) we shall employ a representation which diagonalizes the particle and photon densities; thus, the term $\mathcal{E}$ in $V^{P}$ defines the energies of the states between which particles undergo transitions during collisions. The second term in $V^{P}$ (i.e., $T^{\gamma}$ ) consists of that part of the energy of the photon field which is associated with photon flow between cells as will be seen in a later section. The term $V^{C}$ describes the Coulomb interaction between charged particles in the same cell. One-photon emission and absorption processes are ac- counted for by the term $\mathfrak{g}$, whereas the term $\mathcal{S}$ figures in the calculation of photon-particle scattering. The last term $(x)$ consists of the remainder of $V^{P}$, and to the level of approximation to be considered herein will be ignored henceforth.

If, in Eq. (31), we set $\zeta=I$ and in accordance with the above remarks ignore the terms in $V^{P}$ which contain the explicitly time-dependent external fields, we find that

$$
\Gamma(s)=e^{-i s V^{P} / \hbar} .
$$

The effect of these approximations is to remove environmental influences upon closely associated particles during intervals in which their interaction is to be described in terms of collisions, and, in the present discussion, we will accept the approximations inherent in (49).

In such an event, the transition probability per unit time (42) is conveniently approximated by

$$
\begin{aligned}
& \mathcal{T}_{n^{\prime} n} \simeq(2 \pi / \hbar) \delta\left(E_{n^{\prime}}-E_{n}\right)\left\{\left|V_{n^{\prime} n}{ }^{C}\right|^{2}+\left|\delta_{n^{\prime} n}\right|^{2}\right. \\
& \left.+\left|\sum_{n^{\prime \prime}} \frac{\mathfrak{g}_{n^{\prime} n^{\prime \prime}} V_{n^{\prime \prime} n} C+V_{n^{\prime} n^{\prime \prime}} C_{\mathscr{I}_{n^{\prime \prime} n}}}{E_{n}-E_{n^{\prime \prime}}}\right|^{2}\right\} .
\end{aligned}
$$

Abbreviating the left-hand side of Eq. (43) as $L^{\sigma} f_{\sigma}$, one finds after considerable manipulation that

$$
\begin{aligned}
& L^{\sigma} f_{\sigma}(\mathbf{X}, \mathbf{K}, t)=\sum_{\sigma^{\prime} \mathrm{K}_{1} \mathrm{~K}_{2} \mathrm{~K}_{3}} C_{\sigma \mathrm{K}_{2}, \sigma^{\prime} \mathrm{K}_{3}}^{\sigma \mathrm{K}, \sigma^{\prime} \mathrm{K}_{1}} \sum_{n}\left[n_{\sigma^{\prime} \mathrm{XK}_{3}} n_{\sigma \mathrm{XK}_{2}}\left(1 \pm n_{\sigma \mathrm{XK}}\right)\left(1 \pm n_{\sigma^{\prime} \mathrm{XK}_{1}}\right)-n_{\sigma^{\prime} \mathrm{XK}_{1}} n_{\sigma \mathrm{XK}}\left(1 \pm n_{\sigma \mathrm{XK}_{2}}\right)\left(1 \pm n_{\sigma^{\prime} \mathrm{XK}_{3}}\right)\right] D_{n n} \\
& +\sum_{\mathrm{K}_{1} \lambda \mathrm{k} \lambda^{\prime} \mathbf{k}^{\prime}} S_{\mathrm{K}_{1}, \lambda^{\prime} \mathbf{k}^{\prime}}^{\mathrm{K}, \lambda \mathrm{k}} \sum_{n}\left[n_{\sigma \mathrm{XK}_{1}} n_{\lambda^{\prime} \mathrm{Xk} \mathbf{k}^{\prime}}\left(1 \pm n_{\sigma \mathrm{XK}}\right)\left(1+n_{\lambda \mathrm{Xk}}\right)-n_{\sigma \mathrm{XK}} n_{\lambda \mathrm{Xk}}\left(1 \pm n_{\sigma \mathrm{XK}_{1}}\right)\left(1+n_{\lambda^{\prime} \mathrm{Xk}^{\prime}}\right)\right] D_{n n} \\
& +\sum_{\substack{\sigma^{\prime} \mathbf{K}_{1} \mathbf{K}_{2} \mathbf{K}_{3} n \\
\left(\text { either } \sigma \text { or } \sigma^{\prime}=e\right)}}\left(1-\delta_{\sigma \sigma^{\prime}}\right)\left\{\left[\sum_{\lambda \mathbf{k K}}\left(1+n_{\lambda \mathbf{X} \mathbf{k}}\right) T_{e}\left(\lambda \mathbf{k}, \sigma \mathbf{K}^{\prime}\right)_{\sigma \mathrm{K}_{2}, \sigma^{\prime} \mathbf{K}_{3}}^{\sigma \mathrm{K}, \sigma^{\prime} \mathbf{K}_{1}}+n_{\lambda \mathbf{X k}} T_{a}\left(\lambda \mathbf{k}, \sigma \mathbf{K}^{\prime}\right)_{\sigma \mathbf{K}_{2}, \sigma^{\prime} \mathbf{K}_{3}}^{\sigma \mathrm{K}, \sigma^{\prime} \mathbf{K}_{1}}\right]\right. \\
& \times\left[\left(1-n_{\sigma \mathrm{XK}}\right)\left(1 \pm n_{\sigma^{\prime} \mathrm{XK}_{1}}\right) n_{\sigma \mathrm{XK}_{2}} n_{\sigma^{\prime} \mathrm{XK}_{3}}\right]-\left[\sum_{\lambda \mathbf{k K}}\left(1+n_{\lambda \mathrm{Xk}}\right) T_{e}\left(\lambda \mathbf{k}, \sigma \mathbf{K}^{\prime}\right)_{\sigma \mathrm{K}, \sigma^{\prime} \mathrm{K}_{1}}^{\sigma \mathrm{K}_{2}, \sigma^{\prime} \mathrm{K}_{3}}\right. \\
& \left.\left.+n_{\lambda \times \mathbf{k}} T_{a}\left(\lambda \mathbf{k}, \sigma \mathbf{K}^{\prime}\right)_{\sigma \mathrm{K}, \sigma^{\prime} \mathbf{K}_{1}}^{\sigma \mathbf{K}_{2}, \sigma^{\prime} \mathbf{K}_{3}}\right]\left[\left(1-n_{\sigma \mathrm{XK}_{2}}\right)\left(1 \pm n_{\sigma^{\prime} \mathrm{XK}_{3}}\right) n_{\sigma \mathrm{XK}} n_{\sigma^{\prime} \mathrm{XK}_{1}}\right]\right\} D_{n n}
\end{aligned}
$$

where $C, S, T_{e}$, and $T_{a}$ are essentially the transition probabilities per unit time per unit volume for elastic charged particle scattering, the scattering of charged particles by photons, the scattering of charged particles with photon emissions, and scattering with photon absorption, respectively. The latter two processes have been somewhat specialized in that we have accounted only for photon emission and absorption by electrons when the electrons are scattered by ions. This specialization is not necessary but was made to simplify the balance relation. Actually these inelastic processes make a negligible contribution to the balance relations for the particles when compared to the elastic scattering, but were at least partially included to enable the development of a subsequent $H$ theorem for the system of particles plus photons. The transition probabilities 
represented by $C$ and $S$ are symmetric, i.e.,

$$
\begin{gathered}
C_{\sigma \mathrm{K}_{1}, \sigma^{\prime} \mathbf{K}_{2}}^{\sigma \mathrm{K}, \sigma^{\prime} \mathrm{K}_{3}}=C_{\sigma \mathrm{K}, \sigma^{\prime} \mathrm{K}_{3}}^{\sigma \mathrm{K}_{1}, \sigma^{\prime} \mathbf{K}_{2}}, \\
S_{\mathbf{K}_{1}, \lambda^{\prime} \mathbf{k}^{\prime}}^{\mathbf{K}, \lambda \mathbf{k}}=S_{\mathbf{K}, \lambda \mathbf{k}}^{\mathbf{K}_{1}, \lambda^{\prime} \mathbf{k}^{\prime}} ;
\end{gathered}
$$

whereas the photon emission and absorption transition probabilities are related by

$$
\begin{gathered}
\sum_{\mathbf{K}^{\prime}} T_{a}\left(\lambda \mathbf{k}, \sigma \mathbf{K}^{\prime}\right)_{\sigma \mathbf{K}_{2}, \sigma_{3} \mathbf{K}^{\prime}}^{\sigma \mathbf{K}, \sigma^{\prime} \mathbf{K}_{1}}=T_{a}\left(\lambda \mathbf{k} \mid \sigma \mathbf{K}, \sigma^{\prime} \mathbf{K}_{1} ; \sigma \mathbf{K}_{2}, \sigma^{\prime} \mathbf{K}_{3}\right) \\
=\sum_{\mathbf{K}^{\prime}} T_{e}\left(\lambda \mathbf{k}, \sigma \mathbf{K}^{\prime}\right)_{\sigma \mathbf{K}, \sigma^{\prime} \mathbf{K}_{1}}^{\sigma \mathbf{K}_{2}, \sigma^{\prime} \mathbf{K}_{3}} \\
=T_{e}\left(\lambda \mathbf{k} \mid \sigma \mathbf{K}_{2}, \sigma^{\prime} \mathbf{K}_{3} ; \sigma \mathbf{K}, \sigma^{\prime} \mathbf{K}_{1}\right) .
\end{gathered}
$$

The choice of sign in the factors $(1 \pm n)$ depends upon whether the particles whose number is represented by $n$ are bosons or fermions. These factors appear in our balance relation because of the dependence of reaction rates upon the densities of particles or photons in the final states. In particular, if the particles are fermions (e.g., electrons) so that the factors are of the form $(1-n)$ and the only allowed values of $n$ are 0 and 1 , we see that transitions to occupied states are forbidden as must be the case because of the exclusion principle. However, as indicated earlier, we should not expect this dependence of reaction rates upon the density of par- ticles in final states to be significant in the nondegenerate plasma (a system in which the number of available states greatly exceeds the number of particles).

The direct, formal evaluation of the occupation number sums appearing in (51) leads to the introduction of higher order densities (higher order than the singlet density, e.g., doublet, triplet, and quartet densities) into the balance relation. To circumvent this complication at this point, we resort again to the approximation of replacing averages of products by products of averages - bearing in mind that the average of an $n_{\sigma \mathrm{XK}}$ (or an $n_{\lambda \mathrm{Xk}}$ ) with respect to the density matrix $D$ is just the singlet density for particles of kind $\sigma$ having momentum $\hbar \mathbf{K}$ (or for photons of polarization $\lambda$ and momentum $\hbar \mathbf{k}$ ) multiplied by the cell volume, $L^{3}$. If we further assume that the wavelengths of the particles and photons described by the various distribution functions are very small compared to $L$, we may go to the continuum in momentum space according to

$$
\sum_{\mathbf{K} \in d^{3} K} f_{\sigma}(\mathbf{X}, \mathbf{K}, t)=F_{\sigma}(\mathbf{X}, \mathbf{K}, t) d^{3} K=F_{\sigma}(\mathbf{X}, \mathbf{P}, t) d^{3} P
$$

and

$$
\sum_{\mathbf{k} \in d^{3} k} f_{\lambda}(\mathbf{X}, \mathbf{k}, t)=F_{\lambda}(\mathbf{X}, \mathbf{k}, t) d k d \Omega .
$$

We then find that Eq. (51) may be written as

$$
\begin{aligned}
& L^{\sigma} F_{\sigma}(\mathbf{X}, \mathbf{P}, t)=\sum_{\sigma^{\prime}} \int_{\mathbf{P}_{1} \mathbf{P}_{2} \mathbf{P}_{3}} d^{3} P_{1} d^{3} P_{2} d^{3} P_{3} C\left(\sigma \mathbf{P}_{2}, \sigma^{\prime} \mathbf{P}_{3} ; \sigma \mathbf{P}, \sigma^{\prime} \mathbf{P}_{1}\right) \\
& \times\left\{F_{\sigma}\left(\mathbf{X}, \mathbf{P}_{2}, t\right) F_{\sigma^{\prime}}\left(\mathbf{X}, \mathbf{P}_{3}, t\right)\left[(2 \pi \hbar)^{-3} \pm F_{\sigma}(\mathbf{X}, \mathbf{P}, t)\right]\left[(2 \pi \hbar)^{-3} \pm F_{\sigma^{\prime}}\left(\mathbf{X}, \mathbf{P}_{1}, t\right)\right]\right. \\
& \left.-F_{\sigma}(\mathbf{X}, \mathbf{P}, t) F_{\sigma^{\prime}}\left(\mathbf{X}, \mathbf{P}_{1}, t\right)\left[(2 \pi \hbar)^{-3} \pm F_{\sigma}\left(\mathbf{X}, \mathbf{P}_{2}, t\right)\right]\left[(2 \pi \hbar)^{-3} \pm F_{\sigma^{\prime}}\left(\mathbf{X}, \mathbf{P}_{3}, t\right)\right]\right\} \\
& +\sum_{\lambda \lambda^{\prime}} \int_{\mathbf{P}_{1}, \mathbf{k}, \mathbf{k}^{\prime}} d^{3} P d k d \Omega d k^{\prime} d \Omega^{\prime} S_{\sigma}\left(\mathbf{P}_{1} \lambda^{\prime} \mathbf{k}^{\prime} ; \mathbf{P} \lambda \mathbf{k}\right) \\
& \times\left\{F_{\sigma}\left(\mathbf{X}, \mathbf{P}_{1}, t\right) F_{\lambda^{\prime}}\left(\mathbf{X}, \mathbf{k}^{\prime}, t\right)\left[(2 \pi \hbar)^{-3} \pm F_{\sigma}\left(\mathbf{X}, \mathbf{P}_{1}, t\right)\right]\left[k^{\prime 2}(2 \pi)^{-3}+F_{\lambda^{\prime}}\left(\mathbf{X}, \mathbf{k}^{\prime}, t\right)\right]\right\} \\
& +\sum_{\substack{\sigma^{\prime} \lambda \\
(\text { 入 }}}\left(1-\delta_{\sigma \sigma^{\prime}}\right) \int_{\mathrm{P}_{1} \mathrm{P}_{2} \mathrm{P}_{3}} d^{3} P_{1} d^{3} P_{2} d^{3} P_{3}\left[\int _ { \mathbf { k } } d k d \Omega \left\{\left[k^{2}(2 \pi)^{-3}+F_{\lambda}(\mathbf{X}, \mathbf{k}, t)\right]\right.\right. \\
& \left.\times T_{e}\left(\lambda \mathbf{k} \mid \sigma \mathbf{P}_{,} \sigma^{\prime} \mathbf{P}_{1} ; \sigma \mathbf{P}_{2}, \sigma^{\prime} \mathbf{P}_{3}\right)+F_{\lambda}(\mathbf{X}, \mathbf{k}, t) T_{a}\left(\lambda \mathbf{k} \mid \sigma \mathbf{P}, \sigma^{\prime} \mathbf{P}_{1} ; \sigma \mathbf{P}_{2}, \sigma^{\prime} \mathbf{P}_{3}\right)\right\}\left\{\left[(2 \pi \hbar)^{-3}-F_{\sigma}(\mathbf{X}, \mathbf{P}, l)\right]\right. \\
& \left.\times\left[(2 \pi \hbar)^{-3} \pm F_{\sigma^{\prime}}\left(\mathbf{X}, \mathbf{P}_{1}, t\right)\right] F_{\sigma}\left(\mathbf{X}, \mathbf{P}_{2}, t\right) F_{\sigma^{\prime}}\left(\mathbf{X}, \mathbf{P}_{3}, t\right)\right\}-\int_{\mathbf{k}} d k d \Omega \\
& \times\left\{\left[k^{2}(2 \pi)^{-3}+F_{\lambda}(\mathbf{X}, \mathbf{k}, t)\right] T_{e}\left(\lambda \mathbf{k} \mid \sigma \mathbf{P}_{2}, \sigma^{\prime} \mathbf{P}_{3} ; \sigma \mathbf{P}, \sigma^{\prime} \mathbf{P}_{1}\right)+F_{\lambda}(\mathbf{X}, \mathbf{k}, t) T_{a}\left(\lambda \mathbf{k} \mid \sigma \mathbf{P}_{2}, \sigma^{\prime} \mathbf{P}_{3} ; \sigma \mathbf{P}, \sigma^{\prime} \mathbf{P}_{1}\right)\right\} \\
& \left.\times\left\{\left[(2 \pi \hbar)^{-3}-F_{\sigma}\left(\mathbf{X}, \mathbf{P}_{2}, t\right)\right]\left[(2 \pi \hbar)^{-3} \pm F_{\sigma^{\prime}}\left(\mathbf{X}, \mathbf{P}_{3}, t\right)\right] F_{\sigma}(\mathbf{X}, \mathbf{P}, t) F_{\sigma^{\prime}}\left(\mathbf{X}, \mathbf{P}_{1}, t\right)\right\}\right] \text {. }
\end{aligned}
$$

The transition probability for particle-photon scattering is given by

$$
\begin{aligned}
& S_{\sigma}\left(\mathbf{P}_{1} \lambda^{\prime} \mathbf{k}^{\prime} ; \mathbf{P} \lambda \mathbf{k}\right) \\
& =(2 \pi)^{6} \hbar^{4}\left(\frac{e_{\sigma}^{2}}{m_{\sigma} c}\right)^{2} \frac{c^{2}}{k k^{\prime}}\left|\boldsymbol{\varepsilon}_{\lambda}(\mathbf{k}) \cdot \boldsymbol{\varepsilon}_{\lambda^{\prime}}\left(\mathbf{k}^{\prime}\right)\right|^{2} \\
& \times \delta\left(E_{P}+E_{k}-E_{P_{1}}-E_{k^{\prime}}\right) \delta\left(\mathbf{P}_{1}+\hbar \mathbf{k}^{\prime}-\mathbf{P}-\hbar \mathbf{k}\right) .
\end{aligned}
$$

The transition probabilities for elastic and inelastic charged-particle scattering are not so conveniently expressed in the present instance. However, if one makes the approximation

$$
\begin{aligned}
& L^{-3} \int d^{3} x e^{-i \mathbf{x} \cdot\left(\mathbf{K}-\mathbf{K}^{\prime \prime \prime}+\mathbf{K}^{\prime}-\mathbf{K}^{\prime \prime}\right)} E(\mathbf{X}, \mathbf{x}) \\
& \times \int \frac{d^{3} R}{R} E(\mathbf{X}, \mathbf{x}+\mathbf{R}) e^{-i \mathbf{R} \cdot\left(\mathbf{K}^{\prime}-\mathbf{K}^{\prime \prime}\right)} \\
& \simeq \delta\left(\mathbf{K}-\mathbf{K}^{\prime \prime \prime}+\mathbf{K}^{\prime}-\mathbf{K}^{\prime \prime}\right) \int \frac{d^{3} R}{R} E(\mathbf{X}, \mathbf{R}) e^{-i \mathbf{R} \cdot\left(\mathbf{K}^{\prime}-\mathbf{K}^{\prime \prime}\right)} \\
& \quad=\delta\left(\mathbf{K}-\mathbf{K}^{\prime \prime \prime}+\mathbf{K}^{\prime}-\mathbf{K}^{\prime \prime}\right) U\left(\mathbf{K}^{\prime}-\mathbf{K}^{\prime \prime}\right),
\end{aligned}
$$


formulas for these probabilities may be exhibited as

$$
\begin{aligned}
C\left(\sigma \mathbf{P}_{2}, \sigma^{\prime} \mathbf{P}_{3} ; \sigma \mathbf{P}, \sigma^{\prime} \mathbf{P}_{1}\right) & \\
= & (2 \pi)^{4} \hbar^{2}\left(e_{\sigma} e_{\sigma^{\prime}}\right)^{2}\left|U\left(\mathbf{P}-\mathbf{P}_{2}\right) \pm \delta_{\sigma \sigma^{\prime}} U\left(\mathbf{P}-\mathbf{P}_{3}\right)\right|^{2} \\
& \times \delta\left(E_{P}+E_{P_{1}}-E_{P_{2}}-E_{P_{3}}\right) \delta\left(\mathbf{P}+\mathbf{P}_{1}-\mathbf{P}_{2}-\mathbf{P}_{3}\right),(5
\end{aligned}
$$

and

$$
\begin{aligned}
& T_{e}\left(\lambda \mathbf{k} \mid \sigma \mathbf{P}_{2}, \sigma^{\prime} \mathbf{P}_{3} ; \sigma \mathbf{P}, \sigma^{\prime} \mathbf{P}_{1}\right)=\frac{\pi^{2} \hbar c^{4}}{k}(2 \pi \hbar)^{3} \\
& \times\left(\frac{e_{e}^{2}}{m_{e} c^{2}}\right)^{2}\left(\frac{e_{i}^{2}}{\hbar c}\right)\left|U\left(\mathbf{P}_{1}-\mathbf{P}_{3}\right)\right|^{2} \delta\left(\mathbf{P}+\mathbf{P}_{1}-\mathbf{P}_{2}-\mathbf{P}_{3}-\hbar \mathbf{k}\right) \\
& \times \mid \frac{\mathbf{P}_{2} \cdot \boldsymbol{\varepsilon}_{\lambda}(\mathbf{k})\left\{1-(2 \pi \hbar)^{3} F e\left(\mathbf{P}_{2}+\hbar \mathbf{k}\right)\right\}}{E_{\sigma P}+E_{\sigma^{\prime} P_{1}}-E_{\sigma^{\prime} P_{3}}-E_{\sigma P_{2}+\hbar k}} \\
& +\left.\frac{\mathbf{P} \cdot \boldsymbol{\varepsilon}_{\lambda}(\mathbf{k})\left\{1-(2 \pi \hbar)^{3} F_{e}(\mathbf{P}-\hbar \mathbf{k})\right\}}{E_{\sigma P_{2}}+E_{\sigma^{\prime} P_{3}}-E_{\sigma^{\prime} P_{1}}-E_{\sigma P-\hbar k}}\right|^{2} \\
& \times \delta\left(E_{\sigma P}+E_{\sigma^{\prime} P_{1}}-E_{\sigma P_{2}}-E_{\sigma^{\prime} P_{3}}-E_{k}\right) .
\end{aligned}
$$

The photon-particle scattering frequency consists of the Thomson cross section (as is readily demonstrated by averaging over photon polarization states) and appropriate energy and momentum-conserving delta func- tions. The charged-particle elastic scattering frequency is not quite conventional in that it does not contain the usual Rutherford cross section because of the restrictions on the integrals indicated in Eq. (57). The same remark applies to the inelastic scattering frequency (58b) which, if the indicated restriction is removed and a nondegenerate system is assumed, is the usual nonrelativistic, Born approximation to the bremsstrahlung frequency.

Finally, it is convenient and conventional to express the balance relation (55) in terms of the independent variable $\mathbf{V}$ instead of $\mathbf{P}$. We introduce the velocity variable according to the identification

$$
\mathbf{V}=\left[\mathbf{P}-\left(e_{\sigma} / c\right)\langle\mathbf{R}\rangle\right] / m_{\sigma} .
$$

We further identify the electric and magnetic fields responsible for the smooth accelerations of the particles by

$$
\begin{gathered}
E_{j}=-\frac{\partial \Phi}{\partial X_{j}}-\frac{\partial v}{\partial X_{j}}-\frac{1}{c} \frac{\partial}{\partial t}\left\langle R_{j}\right\rangle, \\
\mathbf{H}=\boldsymbol{\nabla} \times \mathbf{X} \times \mathbf{R}\rangle .
\end{gathered}
$$

Recalling the definition of the operator $L^{\sigma}$, we find that the balance relation becomes

$$
\begin{aligned}
\frac{\partial F_{\sigma}}{\partial t}+ & V_{j} \frac{\partial F_{\sigma}}{\partial X_{j}}+\frac{e_{\sigma}}{m_{\sigma}} E_{j} \frac{\partial F_{\sigma}}{\partial V_{j}}+\frac{e_{\sigma}}{m_{\sigma} c}(\mathbf{V} \times \mathbf{H})_{j} \frac{\partial F_{\sigma}}{\partial V_{j}} \\
= & \sum_{\sigma^{\prime}} \int d^{3} V_{1} d^{3} V_{2} d^{3} V_{3} C\left(\sigma \mathbf{P}_{2, \sigma^{\prime}} \mathbf{P}_{3} ; \sigma \mathbf{P}, \sigma^{\prime} \mathbf{P}_{1}\right)\left[F_{\sigma}\left(\mathbf{V}_{2}\right) F_{\sigma^{\prime}}\left(\mathbf{V}_{3}\right)\left\{\left(\frac{m_{\sigma}}{2 \pi \hbar}\right)^{3} \pm F_{\sigma}(\mathbf{V})\right\}\right. \\
& \left.\times\left\{\left(\frac{m_{\sigma^{\prime}}}{2 \pi \hbar}\right)^{3} \pm F_{\sigma^{\prime}}\left(\mathbf{V}_{1}\right)\right\}-F_{\sigma}(\mathbf{V}) F_{\sigma^{\prime}}\left(\mathbf{V}_{1}\right)\left\{\left(\frac{m_{\sigma}}{2 \pi \hbar}\right)^{3} \pm F_{\sigma}\left(\mathbf{V}_{2}\right)\right\}\left\{\left(\frac{m_{\sigma}}{2 \pi \hbar}\right)^{3} \pm F_{\sigma^{\prime}}\left(\mathbf{V}_{3}\right)\right\}\right] \\
& +\sum_{\lambda \lambda^{\prime}} \int d^{3} V_{1} d k d \Omega d k^{\prime} d \Omega^{\prime} S_{\sigma}\left(\mathbf{P}_{1} \lambda^{\prime} \mathbf{k}^{\prime} ; \mathbf{P} \lambda \mathbf{k}\right)\left[F_{\sigma}\left(\mathbf{V}_{1}\right) F_{\lambda^{\prime}}\left(\mathbf{k}^{\prime}\right)\left\{\left(\frac{m_{\sigma}}{2 \pi \hbar}\right)^{3} \pm F_{\sigma}(\mathbf{V})\right\}\left\{\frac{k^{2}}{(2 \pi)^{3}}+F_{\lambda}(\mathbf{k})\right\}\right. \\
& \left.-F_{\sigma}(\mathbf{V}) F_{\lambda}(\mathbf{k})\left\{\left(\frac{m_{\sigma}}{2 \pi \hbar}\right)^{3} \pm F_{\sigma}\left(\mathbf{V}_{1}\right)\right\}\left\{\frac{k^{\prime 2}}{(2 \pi)^{3}}+F_{\lambda^{\prime}}\left(\mathbf{k}^{\prime}\right)\right\}\right]+\sum_{\sigma^{\prime} \lambda}\left(1-\delta_{\sigma \sigma^{\prime}}\right) \int d^{3} V_{1} d^{3} V_{2} d^{3} V_{3} \\
& \times\left[\int d k d \Omega\left(\left\{\frac{k^{2}}{(2 \pi)^{3}}+F_{\lambda}(\mathbf{k})\right\} T_{e}\left(\lambda \mathbf{k} \mid \sigma \mathbf{P}, \sigma^{\prime} \mathbf{P}_{1} ; \sigma \mathbf{P}_{2}, \sigma^{\prime} \mathbf{P}_{3}\right)+F_{\lambda}(\mathbf{k}) T_{a}\left(\lambda \mathbf{k} \mid \sigma \mathbf{P}, \sigma^{\prime} \mathbf{P}_{1} ; \sigma \mathbf{P}_{2}, \sigma^{\prime} \mathbf{P}_{3}\right)\right)\right. \\
& \times\left(F_{\sigma}\left(\mathbf{V}_{2}\right) F_{\sigma^{\prime}}\left(\mathbf{V}_{3}\right)\left\{\left(\frac{m_{\sigma}}{2 \pi \hbar}\right)^{3}-F_{\sigma}(\mathbf{V})\right\}\left\{\left(\frac{m_{\sigma^{\prime}}}{2 \pi \hbar}\right)^{3} \pm F_{\sigma^{\prime}}\left(\mathbf{V}_{1}\right)\right\}\right)-\int d k d \Omega \\
& \times\left(\left\{\frac{k^{2}}{(2 \pi)^{3}}+F_{\lambda}(\mathbf{k})\right\} T_{e}\left(\lambda \mathbf{k} \mid \sigma \mathbf{P}_{2}, \sigma^{\prime} \mathbf{P}_{3} ; \sigma \mathbf{P}_{, \sigma^{\prime}} \mathbf{P}_{1}\right)+F_{\lambda}(\mathbf{k}) T_{a}\left(\lambda \mathbf{k} \mid \sigma \mathbf{P}_{2}, \sigma^{\prime} \mathbf{P}_{3} ; \sigma \mathbf{P}, \sigma^{\prime} \mathbf{P}_{1}\right)\right) \\
\times & \left.\times\left(F_{\sigma}(\mathbf{V}) F_{\sigma^{\prime}}\left(\mathbf{V}_{1}\right)\left\{\left(\frac{m_{\sigma}}{2 \pi \hbar}\right)^{3}-F_{\sigma}\left(\mathbf{V}_{2}\right)\right\}\left\{\left(\frac{m_{\sigma^{\prime}}}{2 \pi \hbar}\right)^{3} \pm F_{\sigma^{\prime}}\left(\mathbf{V}_{3}\right)\right\}\right)\right]
\end{aligned}
$$

The fields $\mathbf{E}$ and $\mathbf{H}$ appearing in (61) are a superposition of the externally applied and a portion of the internally generated electric and magnetic fields. If we ignore the scattering of particles by photons and the inelastic scattering of particles by particles, and take the classical limit of the terms describing the elastic scattering of 
particles by particles, we find that Eq. (61) is just the conventional Boltzmann equation with self-consistent fields. However, as developed here, this equation seems somewhat hedged by restrictions, which perhaps should be reviewed at this point.

In the present context, these restrictions stem at least partially from the seeming necessity for the operations of spatial and temporal coarse graining. The operation of spatial coarse graining requires that the distribution functions for the particles and the fields $\mathbf{E}$ and $\mathbf{H}$ be essentially constant over an appropriately chosen volume $V$. Thus, given $V$, this puts an obvious limitation on the space rates of change of the fields, whereas given the inhomogeneities of the fields, an upper bound on the dimensions of $V$ is immediately indicated. Furthermore, the distribution functions are expected to represent the average number of particles in the volume $V$ (hence, the particles must be presumed to be localized within $V)$; thus, the least linear dimension of this volume must be large compared to the De Broglie wavelength of the majority of particles of interest. It should be noted that the collision description of the interaction of closely associated particles also requires that their De Broglie wavelengths (actually their relative De Broglie wavelengths) be small compared to any linear measure of $V$. Thus, rapid field variations require a finegrained average, whereas systems of low-mean-energy particles require a coarse-grained average and in some systems these opposing demands may not be met. This is unlikely, however, in the fully ionized plasma. A serious complication is introduced by the presence of strong magnetic fields, even if homogeneous. Evidently, in such a circumstance, it is required that the dimensions of the quantization volume must be small compared to the radius of gyration of the lightest particle of interest in the system. Otherwise, the employment of the plane wave representation for the particles in $V$ would be unsuitable. Finally, the assumption of the localizability of photons in the volume of quantization implies that its least linear dimension be large compared to the wavelengths of such photons.

The equations governing the behavior of $\mathbf{E}$ and $\mathbf{H}$ remain to be developed in the present context. As they represent superpositions of internally generated and externally applied field, and as the external fields are presumed known, it suffices to consider only the portions of $\mathbf{E}$ and $\mathbf{H}$ which arise from charges and currents within the plasma. If we designate these portions by $\mathcal{E}$ and $\mathcal{F}$, respectively, then by Eq. (44) we have

$$
\begin{aligned}
\mathcal{E}_{j}{ }^{L}(\mathbf{X}, t)=\partial v / \partial X_{j} \\
=\frac{\partial}{\partial X_{j}} \sum_{\sigma} e_{\sigma} \frac{d^{3} x[1-E(\mathbf{X}, \mathbf{x})]}{\mid} \\
\qquad \operatorname{x}-\mathbf{X} \mid \\
\times \operatorname{Tr} \psi_{\sigma}^{\dagger}(\mathbf{x}) \psi_{\sigma}(\mathbf{x}) D(t),
\end{aligned}
$$

for the longitudinal part of the self-consistent electric field. For the transverse part of this field we have

$$
\mathcal{E}_{j}{ }^{T}(\mathbf{x}, t)=-\frac{1}{c} \frac{\partial}{\partial t} \operatorname{Tr} A_{j}{ }^{s}(\mathbf{x}) D(t),
$$

whereas for the self-consistent magnetic field, we have

$$
\mathfrak{H}_{j}(\mathbf{x}, t)=\operatorname{Tr}\left\{\boldsymbol{\nabla} \times \mathbf{A}^{s}(\mathbf{x})\right\}_{j} D(t) .
$$

Evidently $\mathfrak{H C}$ is divergenceless; and furthermore, from (63), it is seen that

$$
\begin{aligned}
\boldsymbol{\nabla} \times \mathcal{E}^{T} & =-\frac{1}{c} \frac{\partial}{\partial t} \operatorname{Tr}\left\{\boldsymbol{\nabla} \times \mathbf{A}^{s}\right\} D \\
& =-\frac{1}{c} \frac{\partial}{\partial t} \mathcal{H} C
\end{aligned}
$$

The relations (63), (64), and (65) are essentially identities and enable the calculation of the transverse part of the self-consistent electric field given the corresponding magnetic field. However, the equation relating the fields to the currents in the system does not emerge so readily. The difficulty here seems to stem from the necessity of calculating explicitly operators representing the time derivatives of $\mathbf{A}^{s}$, i.e., of calculating commutators of $\mathbf{A}^{s}$ with the Hamiltonian. But such calculations have here been complicated by the fact that $\mathbf{A}^{\mathbf{s}}$ represents only the "low-frequency" part of the vector potential generated by charges and currents in the plasma. A semi-intuitive circumvention of this difficulty is accomplished by considering an equation satisfied by the exact fields in the plasma, i.e., the fields which have not been decomposed into parts of the "fast" and "slow" variation. Labeling these "complete" fields by $\boldsymbol{\varepsilon}^{\prime}$ and $\mathfrak{H}^{\prime}$ one finds (as shown elsewhere) ${ }^{1}$ that they satisfy the equation,

$$
\boldsymbol{\nabla} \times \mathcal{H}^{\prime}-\frac{1}{c} \frac{\partial}{\partial t} \mathcal{E}^{\prime}=\frac{4 \pi}{c} \operatorname{Tr} \mathbf{J}_{\mathrm{op}} D
$$

where

$$
\mathbf{J}_{\mathrm{op}}=\sum_{\sigma}\left[\frac{i \hbar e_{\sigma}}{2 m_{\sigma}}\left\{\left(\nabla \psi_{\sigma}^{\dagger}\right) \psi_{\sigma}-\psi_{\sigma}^{\dagger} \nabla \psi_{\sigma}\right\}-\frac{e_{\sigma}{ }^{2}}{m_{\sigma} c} \mathbf{A} \psi_{\sigma}{ }^{\dagger} \psi_{\sigma}\right] \text {. }
$$

This is the anticipated relation between the exact fields and the exact currents in the plasma. However, it is desirable to translate the above description of the current to the conventional macroscopic description of plasma currents, i.e.,

$$
\mathbf{J}_{\text {macro }}=\sum_{\sigma} e_{\sigma} \int \mathrm{V} F_{\sigma} d^{3} V
$$

This is readily accomplished, for

$$
\begin{aligned}
\sum_{\sigma} e_{\sigma} \int \mathbf{V} F_{\sigma} d^{3} V & =\sum_{\sigma} \frac{e_{\sigma}}{m_{\sigma}} \int\left(\mathbf{P}-\frac{e_{\sigma}}{c}\langle\mathbf{A}\rangle\right) F_{\sigma} d^{3} P \\
= & \sum_{\sigma} \frac{e_{\sigma} \hbar}{m_{\sigma}} \int \mathbf{K} F_{\sigma} d^{3} K-\sum_{\sigma} \frac{e_{\sigma}{ }^{2}}{m_{\sigma} c}\langle\mathbf{A}\rangle\left\langle\psi_{\sigma}{ }^{\dagger} \psi_{\sigma}\right\rangle,
\end{aligned}
$$


where here of course, $\mathbf{A}$ is interpreted as the total vector potential in the plasma. A little calculation reveals that the first term on the right-hand side of Eq. (69) is the same as the average of the first term on the right-hand side of Eq. (67); so that

$$
\begin{aligned}
\operatorname{Tr} \mathbf{J}_{\mathrm{op}} D \equiv\left\langle\mathbf{J}_{\mathrm{op}}\right\rangle= & \mathbf{J}_{\text {macro }} \\
& +\sum_{\sigma} \frac{e_{\sigma}{ }^{2}}{m_{\sigma} c}\left[\langle\mathbf{A}\rangle\left\langle\psi_{\sigma}^{\dagger} \psi_{\sigma}\right\rangle-\left\langle\mathbf{A} \psi_{\sigma}^{\dagger} \psi_{\sigma}\right\rangle\right] .
\end{aligned}
$$

Entering this relation into Eq. (66), and decomposing $\boldsymbol{\varepsilon}^{\prime}$ and $\mathcal{F}^{\prime}$ into parts of "fast" and "slow" variation we obtain

$$
\begin{aligned}
\boldsymbol{\nabla} \times \mathfrak{H}- & -\frac{1}{c} \frac{\partial}{\partial t} \boldsymbol{E}-\frac{4 \pi}{c} \mathbf{J}_{\text {macro }}+\nabla \times \mathcal{H}^{f}-\frac{1}{c} \frac{\partial}{\partial t} \mathcal{E}^{f} \\
& -\frac{4 \pi}{c} \sum_{\sigma} \frac{e_{\sigma}{ }^{2}}{m_{\sigma} c}\left[\left\langle\mathbf{A}^{f}\right\rangle\left\langle\psi_{\sigma}^{\dagger} \psi_{\sigma}\right\rangle-\left\langle\mathbf{A}^{f} \psi_{\sigma}^{\dagger} \psi_{\sigma}\right\rangle\right]=0 .
\end{aligned}
$$

Note that $\mathbf{A}^{f}$ only enters into the "correlation term" in (71), since we have already employed assumptions equivalent to the statement that

$$
\left\langle\mathbf{A}^{s}\right\rangle\left\langle\psi_{\sigma}{ }^{\dagger} \psi_{\sigma}\right\rangle-\left\langle\mathbf{A}^{s} \psi_{\sigma}{ }^{\dagger} \psi_{\sigma}\right\rangle=0 .
$$

It is at least intuitively reasonable to argue at this point that the macroscopic current $\mathbf{J}_{\text {macro }}$ contributes only to the slowly varying fields $\boldsymbol{E}$ and $\mathfrak{F}$, whereas the "correlation current" contributes only to the rapidly varying component of the internal fields. The latter, however, have already presumably been adequately accounted for in terms of photon distributions; hence, the only relevant part of Eq. (71) is

$$
\nabla \times \mathfrak{H}-\frac{1}{c} \frac{\partial}{\partial t} \boldsymbol{E}=\frac{4 \pi}{c} \mathbf{J}_{\text {macro }}
$$

We now assert that Eqs. (62), (65), and (73) provide an appropriate description of the self-consistent fields appearing in the particle transport Eq. (61).

\section{THE BALANCE RELATIONS FOR THE PHOTON DISTRIBUTIONS}

In order to complete our description of the plasma, we require the equations governing the photon distributions, $F_{\lambda}$. The deduction of these equations for nondispersive media treating spatial coarse-graining in a semi-intuitive fashion has been detailed elsewhere. ${ }^{2}$ Furthermore, a derivation of the photon balance relation in dispersive media employing Ono's method of quantization in cells has also been presented. ${ }^{10}$ Thus, here we concern ourselves mainly with a sketch of the results for the fully ionized plasma in order to facilitate the development of an $\boldsymbol{H}$ theorem in the following section.

The argument here proceeds along lines entirely similar to those outlined above for the particles. Bearing in mind that it is not $T^{\gamma}[\mathrm{Eq} .(47 \mathrm{~b})]$ that governs the transport process, we find in analogy to Eq. (41) that

$$
\begin{gathered}
\left.\frac{f_{\lambda}(\mathbf{X}, \mathbf{k}, t+s)-f_{\lambda}(\mathbf{X}, \mathbf{k}, t)}{s}-\stackrel{i}{i} \underset{\hbar}{-\left[T^{\gamma}, \rho_{\lambda}\right.}(\mathbf{X}, \mathbf{k})\right] D(t) \\
\simeq L^{-3} \sum_{n n^{\prime}}\left(\rho_{\lambda n^{\prime} n^{\prime}}-\rho_{\lambda n n}\right) T_{n^{\prime} n} D_{n n .}
\end{gathered}
$$

In this expression, $\tau_{n^{\prime} n}$ is again suitably approximated by Eq. (50), noting that $V_{n^{\prime} n}^{C}$ will be zero for states differing in their photon occupation numbers, as is indeed the case in the right-hand side of (74). After considerable straightforward calculation, the introduction of various approximations similar to those outlined for the particle case, and making the transition to the continuum-in-momentum space [Eq. (54)] one finds that

$$
\begin{aligned}
& \frac{\partial F_{\lambda}}{\partial t}+c \Omega_{j} \frac{\partial F_{\lambda}}{\partial X_{j}} \\
& =\sum_{\sigma \lambda^{\prime}} \int d^{3} V d^{3} V_{1} d k^{\prime} d \Omega^{\prime} S_{\sigma}\left(\mathbf{P}_{1} \lambda^{\prime} \mathbf{k}^{\prime} ; \mathbf{P} \lambda \mathbf{k}\right)\left[F_{\sigma}\left(\mathbf{V}_{1}\right) F_{\lambda^{\prime}}\left(\mathbf{k}^{\prime}\right)\left\{\left(\frac{m_{\sigma}}{2 \pi \hbar}\right)^{3} \pm F_{\sigma}(\mathbf{V})\right\}\left\{\frac{k^{2}}{(2 \pi)^{3}}+F_{\lambda}(\mathbf{k})\right\}\right. \\
& \left.-F_{\sigma}(\mathbf{V}) F_{\lambda}(\mathbf{k})\left\{\left(\frac{m_{\sigma}}{2 \pi \hbar}\right)^{3} \pm F_{\sigma}\left(\mathbf{V}_{1}\right)\right\}\left\{\frac{k^{\prime 2}}{(2 \pi)^{3}}+F_{\lambda^{\prime}}\left(\mathbf{k}^{\prime}\right)\right\}\right]+\sum_{\sigma \sigma^{\prime}} \frac{\left(1-\delta_{\sigma \sigma^{\prime}}\right)}{2} \int d^{3} V d^{3} V_{1} d^{3} V_{2} d^{3} V_{3} \\
& \times T_{e}\left(\lambda \mathbf{k} \mid \sigma \mathbf{P}, \sigma^{\prime} \mathbf{P}_{1} ; \sigma \mathbf{P}_{2}, \sigma^{\prime} \mathbf{P}_{3}\right)\left[\left\{\frac{k^{2}}{(2 \pi)^{3}}+F_{\lambda}(\mathbf{k})\right\} F_{\sigma}\left(\mathbf{V}_{2}\right) F_{\sigma^{\prime}}\left(\mathbf{V}_{3}\right)\left\{\left(\frac{m_{\sigma}}{2 \pi \hbar}\right)^{3}-F_{\sigma}(\mathbf{V})\right\}\left\{\left(\frac{m_{\sigma^{\prime}}}{2 \pi \hbar}\right)^{3} \pm F_{\sigma^{\prime}}\left(\mathbf{V}_{1}\right)\right\}\right] \\
& -\sum_{\sigma \sigma^{\prime}} \frac{\left(1-\delta_{\sigma \sigma^{\prime}}\right)}{2} \int d^{3} V d^{3} V_{1} d^{3} V_{2} d^{3} V_{3} T_{a}\left(\lambda \mathbf{k} \mid \sigma \mathbf{P}_{2}, \sigma^{\prime} \mathbf{P}_{3} ; \sigma \mathbf{P}, \sigma^{\prime} \mathbf{P}_{1}\right)\left[F_{\lambda}(\mathbf{k}) F_{\sigma}(\mathbf{V}) F_{\sigma^{\prime}}\left(\mathbf{V}_{1}\right)\left\{\left(\frac{m_{\sigma}}{2 \pi \hbar}\right)^{3}-F_{\sigma}\left(\mathbf{V}_{2}\right)\right\}\right. \\
& \left.\times\left\{\left(\frac{m_{\sigma^{\prime}}}{2 \pi \hbar}\right)^{3} \pm F_{\sigma^{\prime}}\left(\mathbf{V}_{3}\right)\right\}\right] .
\end{aligned}
$$


This equation is substantially the same as the portions of Eq. (47) in reference 2 which are relevant to the fully ionized plasma, except for the absence here of the influence of cyclotron radiation on the photon distribution. This deficiency arises because of the difficulty of using anything but momentum states for the particles in the context of the cell quantization procedure. Though one surmises that the difficulty could be circumvented (at least in a useful, approximate sense) by the employment of arguments similar to those advanced in reference 2, the attempt to do so will not be presented here. Thus, the issue of developing a consistent treatment of particle and photon transport taking account of collisions in the presence of strong magnetic fields will be left unresolved for the time being. Note also that the present discussion takes no account of the dispersive effects of the medium on photon transport. These effects have been studied extensively in reference 10 .

\section{THERMODYNAMICS OF THE FULLY IONIZED PLASMA}

The present description of the plasma is complete, irreversible (in the sense of the many and varied approximations that have been introduced into its development), and consistent. It is complete in the sense that we have as many equations as we have unknown functions described by them, and these equations involve functional parameters (scattering frequencies, emission and absorption probabilities, etc.) whose analytical representations have been specified. It is irreversible in the sense that the whole set is not invariant under the transformation

$$
(\mathbf{V}, \mathbf{k}, \mathbf{H}, t) \rightarrow(-\mathbf{V},-\mathbf{k},-\mathbf{H},-t)
$$

Thus, this description of the plasma should contain the important implication that, under certain circumstances at least, the system progresses irreversibly to a unique state referred to as the thermodynamic state; and that in that state the system variables assume specified forms. It is consistent in the sense that the equations explicitly describe the full effect of any given interaction upon all distributions to the same level of approximation. It was to achieve consistency in this sense that led to the inclusion of the effects of inelastic charged particle scattering in the particle balance relations. The quantitative significance of these interactions for the description of the fully ionized plasma is most probably negligible. But to achieve a proper qualitative appreciation of the approach to equilibrium, the influence of these kinds of interaction between particles and photons on the particle distributions cannot be ignored.

We now undertake the task of proving an $H$ theorem for systems that are not exposed to external fields, that have already become spatially homogeneous, that exhibit neither internal electric currents nor space charge, but that are still temporally varying. To this end we define a function $S$ according to ${ }^{11}$

$$
\begin{aligned}
S= & -\kappa \sum_{\sigma} \int d^{3} V\left\{F_{\sigma}(\mathbf{V}) \ln F_{\sigma}(\mathbf{V}) \pm r_{\sigma} \ln r_{\sigma}\right. \\
& \left.\mp\left[r_{\sigma} \pm F_{\sigma}(\mathbf{V})\right] \ln \left[r_{\sigma} \pm F_{\sigma}(\mathbf{V})\right]\right\} \\
& -\kappa \sum_{\lambda} \int d k d \Omega\left\{F_{\lambda}(\mathbf{k}) \ln F_{\lambda}(\mathbf{k})+r_{\lambda} \ln r_{\lambda}\right. \\
& \left.-\left[r_{\lambda}+F_{\lambda}(\mathbf{k})\right] \ln \left[r_{\lambda}+F_{\lambda}(\mathbf{k})\right]\right\} .
\end{aligned}
$$

It is then readily shown that

$$
\begin{aligned}
\frac{d S}{d t}=-\kappa \sum_{\sigma} \int d^{3} V & \ln \left\{\frac{F_{\sigma}(\mathbf{V})}{r_{\sigma} \pm F_{\sigma}(\mathbf{V})}\right\} \frac{\partial F_{\sigma}}{\partial t} \\
& -\kappa \sum_{\lambda} \int d k d \Omega \ln \left\{\frac{F_{\lambda}(\mathbf{k})}{r_{\lambda}+F_{\lambda}(\mathbf{k})}\right\} \frac{\partial F_{\lambda}}{\partial t} .
\end{aligned}
$$

In these equations we have introduced, for compactness, the symbols, $\boldsymbol{r}_{\sigma}=\left(m_{\sigma} / 2 \pi \hbar\right)^{3}$ and $r_{\lambda}=k^{2} /(2 \pi)^{3}$. Employing Eqs. (61) and (75) to eliminate the derivatives of the distribution functions in (77), we find

$$
\begin{aligned}
\frac{d S}{d t}= & -\frac{\kappa}{4} \sum_{\sigma=e, i} d^{3} V d^{3} V_{1} d^{3} V_{2} d^{3} V_{3} C\left(\sigma \mathbf{P}_{2}, \sigma \mathbf{P}_{3} ; \sigma \mathbf{P}, \sigma \mathbf{P}_{1}\right)\left[r_{\sigma} \pm F_{\sigma}(\mathbf{V})\right]\left[r_{\sigma} \pm F_{\sigma}\left(\mathbf{V}_{1}\right)\right] \\
& \times\left[r_{\sigma} \pm F_{\sigma}\left(\mathbf{V}_{2}\right)\right]\left[r_{\sigma} \pm F_{\sigma}\left(\mathbf{V}_{3}\right)\right] \ln \left\{\frac{F_{\sigma}(\mathbf{V}) F_{\sigma}\left(\mathbf{V}_{1}\right)}{\left[r_{\sigma} \pm F_{\sigma}(\mathbf{V})\right]\left[r_{\sigma} \pm F_{\sigma}\left(\mathbf{V}_{1}\right)\right]} \frac{\left[r_{\sigma} \pm F_{\sigma}\left(\mathbf{V}_{2}\right)\right]\left[r_{\sigma} \pm F_{\sigma}\left(\mathbf{V}_{3}\right)\right]}{F_{\sigma}\left(\mathbf{V}_{2}\right) F_{\sigma}\left(\mathbf{V}_{3}\right)}\right\} \\
& \times\left\{\frac{F_{\sigma}\left(\mathbf{V}_{2}\right) F_{\sigma}\left(\mathbf{V}_{3}\right)}{\left[r_{\sigma} \pm F_{\sigma}\left(\mathbf{V}_{2}\right)\right]\left[r_{\sigma} \pm F_{\sigma}\left(\mathbf{V}_{3}\right)\right]}-\frac{F_{\sigma}(\mathbf{V}) F_{\sigma}\left(\mathbf{V}_{1}\right)}{\left[r_{\sigma} \pm F_{\sigma}(\mathbf{V})\right]\left[r_{\sigma} \pm F_{\sigma}\left(\mathbf{V}_{1}\right)\right]}\right\}-\frac{\kappa}{2} \int d^{3} V d^{3} V_{1} d^{3} V_{2} d^{3} V_{3} \\
& \times C\left(e \mathbf{P}_{2}, i \mathbf{P}_{3} ; e \mathbf{P}, i \mathbf{P}_{1}\right)\left[r_{e}-F_{e}(\mathbf{V})\right]\left[r_{i} \pm F_{i}\left(\mathbf{V}_{1}\right)\right]\left[r_{e}-F_{e}\left(\mathbf{V}_{2}\right)\right]\left[r_{i} \pm F_{i}\left(\mathbf{V}_{3}\right)\right] \\
& \times \ln \left\{\frac{F_{e}(\mathbf{V}) F_{i}\left(\mathbf{V}_{1}\right)}{\left[r_{e}-F_{e}(\mathbf{V})\right]\left[r_{i} \pm F_{i}\left(\mathbf{V}_{1}\right)\right]} \frac{\left[r_{e}-F_{e}\left(\mathbf{V}_{2}\right)\right]\left[r_{i} \pm F_{i}\left(\mathbf{V}_{3}\right)\right]}{F_{e}\left(\mathbf{V}_{2}\right) F_{i}\left(\mathbf{V}_{3}\right)}\right\}
\end{aligned}
$$

${ }^{11}$ J. E. Mayer and M. G. Mayer, Statistical Mechanics (John Wiley \& Sons, Inc., New York, 1940). 


$$
\begin{aligned}
& \times\left\{\frac{F_{e}\left(\mathbf{V}_{2}\right) F_{i}\left(\mathbf{V}_{3}\right)}{\left[r_{e}-F_{e}\left(\mathbf{V}_{2}\right)\right]\left[r_{i} \pm F_{i}\left(\mathbf{V}_{3}\right)\right]}-\frac{F_{e}(\mathbf{V}) F_{i}\left(\mathbf{V}_{1}\right)}{\left[r_{e}-F_{e}(\mathbf{V})\right]\left[r_{i} \pm F_{i}\left(\mathbf{V}_{1}\right)\right]}\right\}-\kappa \sum_{\sigma \lambda \lambda^{\prime}} \int d^{3} V d^{3} V_{1} d k d \Omega d k^{\prime} d \Omega^{\prime} \\
& \times S_{\sigma}\left(\mathbf{P}_{1} \lambda^{\prime} \mathbf{k}^{\prime} ; \mathbf{P} \lambda \mathbf{k}\right)\left[r_{\sigma} \pm F_{\sigma}(\mathbf{V})\right]\left[r_{\sigma} \pm F_{\sigma}\left(\mathbf{V}_{1}\right)\right]\left[r_{\lambda}+F_{\lambda}(\mathbf{k})\right]\left[r_{\lambda^{\prime}}+F_{\lambda^{\prime}}\left(\mathbf{k}^{\prime}\right)\right] \\
& \times \ln \left\{\frac{F_{\sigma}(\mathbf{V}) F_{\lambda}(\mathbf{k})}{\left[r_{\sigma} \pm F_{\sigma}(\mathbf{V})\right]\left[r_{\lambda}+F_{\lambda}(\mathbf{k})\right]} \frac{\left[r_{\sigma} \pm F_{\sigma}\left(\mathbf{V}_{1}\right)\right]\left[r_{\lambda^{\prime}}+F_{\lambda^{\prime}}\left(\mathbf{k}^{\prime}\right)\right]}{F_{\sigma}\left(\mathbf{V}_{1}\right) F_{\lambda^{\prime}}\left(\mathbf{k}^{\prime}\right)}\right\} \\
& \left.\times\left\{\frac{F_{\sigma}\left(\mathbf{V}_{1}\right) F_{\lambda^{\prime}}\left(\mathbf{k}^{\prime}\right)}{\left[r_{\sigma} \pm F_{\sigma}\left(\mathbf{V}_{1}\right)\right]\left[r_{\lambda^{\prime}}+F_{\lambda^{\prime}}\left(\mathbf{k}^{\prime}\right)\right]}-\frac{F_{\sigma}(\mathbf{V}) F_{\lambda}(\mathbf{k})}{\left[r_{\sigma} \pm F_{\sigma}(\mathbf{V})\right]\left[r_{\lambda}+F_{\lambda}(\mathbf{k})\right]}\right\}-\kappa \sum_{\lambda}\right\} d^{3} V d^{3} V_{1} d^{3} V_{2} d^{3} V_{3} d k d \Omega \\
& \times T_{e}\left(\lambda \mathbf{k} \mid e \mathbf{P}, i \mathbf{P}_{1} ; e \mathbf{P}_{2}, i \mathbf{P}_{3}\right)\left[r_{e}-F_{e}(\mathbf{V})\right]\left[r_{i} \pm F_{i}\left(\mathbf{V}_{1}\right)\right]\left[r_{e}-F_{e}\left(\mathbf{V}_{2}\right)\right]\left[r_{i} \pm F_{i}\left(\mathbf{V}_{3}\right)\right]\left[r_{\lambda}+F_{\lambda}(\mathbf{k})\right] \\
& \times \ln \left\{\frac{F_{e}\left(\mathbf{V}_{1}\right) F_{i}\left(\mathbf{V}_{1}\right) F_{\lambda}(\mathbf{k})}{\left[r_{e}-F_{e}(\mathbf{V})\right]\left[r_{i}+F_{i}\left(\mathbf{V}_{1}\right)\right]\left[r_{\lambda}+F_{\lambda}(\mathbf{k})\right]} \frac{\left[r_{e}-F_{e}\left(\mathbf{V}_{2}\right)\right]\left[r_{i} \pm F_{i}\left(\mathbf{V}_{3}\right)\right]}{F_{e}\left(\mathbf{V}_{2}\right) F_{i}\left(\mathbf{V}_{3}\right)}\right\} \\
& \times\left\{\frac{F_{e}\left(\mathbf{V}_{2}\right) F_{i}\left(\mathbf{V}_{3}\right)}{\left[r_{e}-F_{e}\left(\mathbf{V}_{2}\right)\right]\left[r_{i} \pm F_{i}\left(\mathbf{V}_{3}\right)\right]}-\frac{\left[r_{e}-F_{e}(\mathbf{V})\right]\left[r_{i} \pm F_{i}\left(\mathbf{V}_{1}\right)\right]\left[r_{\lambda}+F_{\lambda}(\mathbf{k})\right]}{\left[\mathbf{V}_{1}\right) F_{\lambda}(\mathbf{k})}\right.
\end{aligned}
$$

In this expression we have assumed a plasma consisting of electrons and only one type of ion. The fact that electrons are fermions and photons are bosons has been accounted for explicitly where suitable, whereas the possibility of either kind of ion has been allowed for. Maximum use has been made of the symmetries inherent in the scattering, emission and absorption frequencies. The contributions to $\dot{S}$ arising from the scattering of particles by particles are the quantum generalization of those presented in the classical development of an $H$ theorem for the two-component, neutral, ideal gas ${ }^{12}$; and the contributions arising from the scattering of particles by photons are the anticipated analogs thereof. The remaining terms are contributed by processes involving the emission and absorption of photons. It was for the purpose of guaranteeing the proper structure of these last contributions to $\dot{S}$ that the effects of inelastic particle scattering were incorporated into the particle balance relation. Since $r_{e}-F_{\epsilon}$ $\geqslant 0$ (the density of Fermions can never exceed the density of available states), it is evident that $\dot{S} \geqslant 0$ always, and equal to zero only if each and every integrand in Eq. (78) separately vanishes. The conclusions to be drawn from the vanishing of the several integrands can be summarized by consideration of the last one. In this instance we require

$$
\begin{array}{r}
\ln \left\{\frac{F_{e}(\mathbf{V})}{r_{e}-F_{e}(\mathbf{V})}\right\}+\ln \left\{\frac{F_{i}\left(\mathbf{V}_{1}\right)}{r_{i} \pm F_{i}\left(\mathbf{V}_{1}\right)}\right\}+\ln \left\{\frac{F_{\lambda}(\mathbf{k})}{r_{\lambda}+F_{\lambda}(\mathbf{k})}\right\} \\
=\ln \left\{\frac{F_{e}\left(\mathbf{V}_{2}\right)}{r_{e}-F_{e}\left(\mathbf{V}_{2}\right)}\right\}+\ln \left\{\frac{F_{i}\left(\mathbf{V}_{3}\right)}{r_{i} \pm F_{i}\left(\mathbf{V}_{3}\right)}\right\} .
\end{array}
$$

At the same time we note that the emission frequency

12 Sydney Chapman and T. G. Cowling, The Mathematical Theory of Non-Uniform Gases (Cambridge University Press, Cambridge, England, 1952).
$T_{e}$ incorporates the conservation rules,

and

$$
E_{e \mathbf{V}}+E_{i \mathbf{V}_{1}}+E_{\mathrm{k}}=E_{e \mathbf{V}_{2}}+E_{i \mathbf{V}_{3}},
$$

$$
m_{e} \mathbf{V}+m_{i} \mathbf{V}_{1}+\hbar \mathbf{k}=m_{e} \mathbf{V}_{2}+m_{i} \mathbf{V}_{3} .
$$

From Eqs. (79) and (80) we may conclude that a general form for the distributions characteristic of the state of the system for which $\dot{S}=0$ is

$$
\begin{aligned}
F_{e}(\mathbf{V}) & =\frac{\left(m_{e} / 2 \pi \hbar\right)^{3}}{\exp \left\{\beta\left[\mu_{e}+m_{e}(\mathbf{V}-\mathbf{W})^{2} / 2\right]\right\}+1} \\
F_{i}\left(\mathbf{V}_{1}\right) & =\frac{\left(m_{i} / 2 \pi \hbar\right)^{3}}{\exp \left\{\beta\left[\mu_{i}+m_{i}\left(\mathbf{V}_{1}-\mathbf{W}\right)^{2} / 2\right]\right\} \mp 1}, \\
F_{\lambda}(\mathbf{k}) & =\frac{k^{2} /(2 \pi)^{3}}{\exp \left\{\beta\left[E_{n}-\hbar \mathbf{k} \cdot \mathbf{W}\right]\right\}-1} .
\end{aligned}
$$

In line with the remarks in Sec. II relative to Eq. (47a), we here take the photon energy to be

$$
E_{k}=\hbar c k\left[1+\sum_{\sigma} \frac{2 \pi e_{\sigma}^{2} \rho_{\sigma}(\mathbf{X})}{m_{\sigma} c^{2} k^{2} L^{3}}\right] .
$$

The parameters $\mu_{e}, \mu_{i}, \beta$, and $\mathbf{W}$ are not determined by the sole requirement that $\dot{S}=0$. However, the fact that they must be independent of position and time (because the distributions must be independent of space and time at this point in the present argument) suggests that the only interpretation available to $\mathbf{W}$ is that of an irrelevant, over-all velocity of the systemhence, here it might as well be taken to be zero. The further fact that we are dealing with the fully ionized, nonrelativistic plasma suggests that $\mu_{e}$ and $\mu_{i}$ be determined by the requirement that the integrals of the particle distributions over velocity space represent the 
constant densities appropriate to the system. And finally, the desire to interpret $S$ as the system entropy suggests the identification of $\beta$ (the only parameter now common to all of the distributions) as $(\kappa T)^{-1}$, where $\kappa$ is taken to be Boltzmann's constant. With these interpretations and identifications, it is evident that the distributions $(81 \mathrm{a}, \mathrm{b}, \mathrm{c})$ are the conventional ones for an equilibrium gas mixture of Fermi-and Boseparticles and photons.

\section{DISCUSSION}

Equation (61) for the particle balance relations and Eqs. (62), (65), and (71) for the self-consistent electromagnetic fields represent a simultaneous, formal generalization and restriction on the usual BoltzmannVlasov-Maxwell description of the fully ionized plasma. They are generalizations in that for the particle balance relations the quantum statistics for the particles have been accounted for as well as the influence of particlephoton scattering and inelastic (radiative) particleparticle scattering, whereas for the field equations the presence of "fluctuation" or "correlation" currents has been noted. They represent restrictions in that the variability of the distributions and self-consistent (as well as external) fields in space, time, and frequency has been limited by the necessity for coarse-graining in space and time. If both the generalizations and the restrictions are ignored, it is seen that the equations comprise the conventional description. It should be noted of course that, if one wishes to ignore "collision" effects entirely, the Vlasov equation for a non-coarsegrained particle distribution is derivable in the classical limit, subject only to the approximations of replacing multiplet densities by products of singlet densities.

The generalizations noted here are not anticipated to be of much significance in the study of terrestial plasmas, and, as has been evident throughout, the restrictions have merely been qualitatively noted and not quantitatively evaluated. Thus, it is our opinion that the importance of this work stems from the demonstration that the extensively employed Boltzmann-VlasovMaxwell description of the fully ionized plasma is logically deducible in some sense, and from the sketching of a framework in which the restrictions on such a description can be investigated in some detail.

\section{ACKNOWLEDGMENT}

The author is grateful to the Michigan MemorialPhoenix Project of The University of Michigan for a grant supporting the preparation of this paper. 